\title{
WIYN Imaging of the Globular Cluster Systems of the Spiral Galaxies NGC 891 and NGC 4013
}

\author{
Katherine L. Rhode, Jessica L. Windschitl, and Michael D. Young \\ Department of Astronomy, Indiana University, Swain West 319, 727 East Third Street, \\ Bloomington, IN 47405-7105,USA; rhode@astro.indiana.edu
}

\begin{abstract}
We present results from a WIYN 3.5-m telescope imaging study of the globular cluster (GC) systems of the edge-on spiral galaxies NGC 891 and NGC 4013. We used the $10^{\prime} \times 10^{\prime}$ Minimosaic Imager to observe the galaxies in $B V R$ filters to projected radii of $\sim 20 \mathrm{kpc}$ from the galaxy centers. We combined the WIYN data with archival and published data from the WFPC2 and Advanced Camera for Surveys on the Hubble Space Telescope to assess the contamination level of the WIYN GC candidate sample and to follow the GC systems further in toward the galaxies' centers. We constructed radial distributions for the GC systems using both the WIYN and HST data. The GC systems of NGC 891 and NGC 4013 extend to $9 \pm 3 \mathrm{kpc}$ and $14 \pm 5 \mathrm{kpc}$, respectively, before falling off to undetectable levels in our images. We use the radial distributions to calculate global values for the total number $\left(N_{G C}\right)$ and specific frequencies $\left(S_{N}\right.$ and $\left.T\right)$ of GCs. NGC 4013 has $N_{G C}=140 \pm 20, S_{N}=1.0 \pm 0.2$ and $T=1.9 \pm 0.5$; our $N_{G C}$ value is $\sim 40 \%$ smaller than a previous determination from the literature. The HST data were especially useful for NGC 891, because the GC system is concentrated toward the plane of the galaxy and was only weakly detected in our WIYN images. Although NGC 891 is thought to resemble the Milky Way in terms of its overall properties, it has only half as many GCs, with $N_{G C}=70 \pm 20, S_{N}=0.3 \pm 0.1$ and $T=0.6 \pm 0.3$. We also calculate the galaxy-mass-normalized number of blue (metal-poor) GCs in NGC 891 and NGC 4013 and find that they fall along a general trend of increasing specific frequency of blue GCs with increasing galaxy mass. Given currently available resources, the optimal method for studying the global properties of extragalactic GC systems is to combine HST data with widefield, ground-based imaging with good resolution. The results here demonstrate the advantage gained by using both methods when possible.
\end{abstract}

Subject headings: galaxies: formation — galaxies: individual (NGC 4013, NGC 891) - galaxies: spiral — galaxies: star clusters: general 


\section{Introduction}

Globular clusters (GCs) are among the most useful stellar populations to study in galaxies. These compact star clusters each contain $\sim 10^{3}-10^{6}$ of stars that appear to have formed over a relatively short period of time and under similar conditions (e.g., Ashman \& Zepf 1992; Ashman \& Zepf 1998; West et al. 2004; Brodie \& Strader 2006; Griffen et al. 2009; Gnedin 2010). GCs are luminous (Milky Way GCs typically have $M_{V} \sim-10$ to -5 ; Harris 1996), often many Gyr old (e.g., Chaboyer et al. 1998, Brodie \& Strader 2006), appear to be associated with major star formation episodes in galaxies (Brodie \& Strader 2006, Bastian 2008), and may be forming in nearby galaxy mergers and/or starbursts (e.g., Whitmore \& Schweizer 1995, de Grijs et al. 2001). GCs are detected in virtually all giant galaxies that have been imaged to sufficient depth to cover a significant fraction of the GC luminosity function (GCLF; Harris 1991, Brodie \& Strader 2006). Together, these properties mean that GCs can be used as a stellar record of the major events associated with the origin and evolution of the galaxies that host them. The numbers, spatial distributions, luminosities, metallicities, and kinematics of the GCs provide fundamental information about the star formation and assembly histories of giant galaxies (Brodie \& Strader 2006).

Motivated primarily by these ideas, we have been carrying out a wide-field CCD imaging survey of the GC systems of giant spiral, elliptical, and S0 galaxies in the local universe (distances to $\sim 20 \mathrm{Mpc}$ ). The spiral galaxies we target are all edge-on or nearly edge-on, to make it easier to identify bona fide GCs and less likely that we will mistake knots of star formation or open clusters in the galaxy disk for GCs. We use large-format and mosaic CCD detectors to image the target galaxies in multiple broadband filters and select as GC candidates the point sources around the galaxies with the expected magnitudes and colors. We use the GC candidate lists and galaxy images to estimate the global properties of each galaxy's GC system - namely, the total number and specific frequency of GCs as well as the spatial and color distribution of the overall system. Since GC systems of nearby galaxies are extended (often covering tens of arc minutes from the galaxy center), wide-field imaging is necessary to cover enough of the GC system to allow for direct, accurate determinations of these quantities.

The general goal of the survey is to increase the number of galaxies for which accurate measurements of the global GC system properties exist, so that these measurements can in turn be used to test scenarios for the formation and evolution of giant galaxies. The details of the design and methods of the survey, as well as the results from the first ten galaxies studied, are given in Rhode \& Zepf (2001, 2003, 2004; hereafter RZ01, RZ03, RZ04) and in Rhode et al. (2005, 2007; hereafter R05, R07). Another motivation for the survey is to use the GC candidate lists as target lists for follow-up spectroscopy, in order to measure 
the radial velocities of the GCs around each galaxy. The kinematics of the GCs provide additional insight into the origin of each host galaxy and the radial velocities can also be used to quantify the galaxy's mass distribution (e.g., Bergond et al. 2006, Bridges et al. 2007).

In this paper, we present results for two more galaxies imaged as part of the widefield survey: NGC 4013 and NGC 891, both edge-on Sb spiral galaxies. Some fundamental properties of the galaxies are summarized in Table1. To analyze these galaxies' GC systems, we combined wide-field mosaic CCD data obtained with the WIYN 3.5-m telescope 1 with previously published results from Hubble Space Telescope (HST) imaging studies of the inner parts of the galaxies' GC systems. Ground-based imaging and HST imaging each have advantages and disadvantages as methods for studying extragalactic GC systems. Groundbased imaging with large-format CCDs often enables one to image all, or nearly all, of the galaxy's GC system in a single pointing. However, limited resolution makes it difficult to distinguish GCs from distant background galaxies and to detect GCs close in to the center of the parent galaxy, especially in the case of a spiral galaxy with a dusty disk and/or active regions of star formation. HST's superb resolution allows one to detect GCs further in toward the centers of their host galaxies, to distinguish GCs from compact background galaxies, and in some cases to study the sizes and structural parameters of the individual GCs (e.g., Spitler et al. 2006, Forbes et al. 2010, Harris et al. 2010). On the other hand, the limited fields-of-view of the HST imagers means that only a fraction of the GC system is typically observed (see discussions in Ashman \& Zepf 1998 and Brodie \& Strader 2006), and properties such as the overall spatial distribution and specific frequency of the GC system can be undetermined or highly uncertain without supplemental data or assumptions (e.g., Goudfrooij et al. 2003, Chandar, Whitmore, \& Lee 2004, Peng et al. 2008, Cantiello, Brocato, \& Blakeslee 2009).

The results presented here for NGC 4013 and NGC 891 demonstrate the efficacy of combining wide-field ground-based data with data from HST to study GC systems of giant galaxies in the local universe. The following section describes the WIYN observations and initial image reduction steps. Section 3 presents the techniques used to detect and select GC candidates in the WIYN images. Section 4 describes additional analysis steps we performed to assess the completeness and contamination level of the WIYN data and ends with a description of how we combined the published HST results for these galaxies with the WIYN data. The overall results for the galaxies' GC systems are described in Section 5. The paper ends with a summary of our main conclusions in Section 6.

\footnotetext{
${ }^{1}$ The WIYN Observatory is a joint facility of the University of Wisconsin, Indiana University, Yale University, and the National Optical Astronomy Observatory.
} 


\section{Observations and Image Reductions}

NGC 891 and NGC 4013 were imaged in three broadband filters $(B V R)$ with the Minimosaic camera on the 3.5-m WIYN telescope at Kitt Peak National Observatory. The WIYN Minimosaic is constructed of two 2048x4096-pixel CCDs with 0.14" pixels and the field-ofview is $9.6^{\prime}$ on each side. NGC 891 is relatively nearby (see Table 1) and thus was positioned on the edge of the detector to maximize the radial coverage of its GC system; NGC 4013 is more distant and was placed in the center of the field. Figures 11 and 2 show the locations of the WIYN fields, superposed on gray-scale images from the Digitized Sky Survey2. The WIYN observations analyzed for this paper were acquired in 2001 January and 2009 March; Table 2 lists the dates, number of images, and exposure times used for each galaxy pointing.

The sky conditions were photometric during the second night of the 2001 January observing run. On that night, images of standard stars from Landolt (1992) were taken, along with one or more exposures of NGC 891 and NGC 4013, in all three filters. A set of photometric calibration coefficients (color terms and zero point constants) was derived using the Landolt star observations taken that night. The color coefficient in the $V$ magnitude equation was $0.02 \pm 0.01$. The color coefficients in the $B-V$ and $V-R$ color equations were $1.02 \pm 0.01$ and $1.05 \pm 0.03$, respectively. The formal errors on the zero points in the $V$, $B-V$ and $V-R$ calibration equations ranged from 0.004 to 0.006 , confirming that the sky conditions that night were indeed photometric.

Images of the target galaxies taken on other, non-photometric nights during the 2001 January run were calibrated by scaling them to a selected image taken on the photometric night and then combining them with that image (see below for details of the combining process). For NGC 4013, a set of $V$ images was obtained in 2009 March; those images were postcalibrated by calculating the zero-point offset in $V$ magnitude between the final, combined 2009 March $V$ image and a single $V$ image taken in 2001 January.

Initial reductions (overscan and bias level subtraction, flat-field division) were performed on the Minimosaic images using standard reduction tasks in the IRAF package MSCRED. The tasks msczero, msccmatch, and mscimage were then used to transform the multiextension FITS images into single-extension images. All of the images for a given galaxy were aligned to match a single pointing. A constant sky background level was computed and then subtracted from each individual galaxy exposure. Images of the same galaxy target,

\footnotetext{
${ }^{2}$ The Digitized Sky Surveys were produced at the Space Telescope Science Institute under U.S. Government grant NAG W-2166.The images of these surveys are based on photographic data obtained using the Oschin Schmidt Telescope on Palomar Mountain and the UK Schmidt Telescope. The plates were processed into compressed digital form with the permission of these institutions.
} 
taken in the same filter, were scaled to a common flux level and combined using the IRAF task imcombine and the ccdclip pixel rejection algorithm. The constant sky background level measured in the image used as a reference in the scaling step was added to the final combined image. The end result was one deep, stacked image per filter for each galaxy target. The mean full-width at half-maximum of the point-spread function (FWHM PSF) in the final combined images ranges from $1.0^{\prime \prime}-1.2^{\prime \prime}$ for NGC 891 and $0.7^{\prime \prime}-0.8^{\prime \prime}$ for NGC 4013.

\section{Detection and Analysis of the Globular Cluster System}

\subsection{Source Detection}

GCs should appear in our ground-based WIYN Minimosaic images as a slight overdensity of point sources surrounding the target galaxy. To detect and then analyze the GC systems of NGC 891 and NGC 4013, we executed the same series of steps used in the previous studies that are part of our wide-field survey (RZ01, RZ03, RZ04, R07). We first smoothed the combined images using a circular median filter with a diameter equal to $\sim 7.25-7.5$ times the mean FWHM PSF of the image. The smoothed images were subtracted from the original versions to remove the diffuse light from the target galaxy and make it easier to locate the point-source GCs. The constant sky background level present in the original images was added to the galaxy-subtracted images; all subsequent analysis steps were performed on the galaxy-subtracted images. The IRAF task daofind was used to detect sources above a given signal-to-noise level in each frame. Areas of the images likely to produce spurious source detections - i.e., near saturated stars and in the dust-obscured spiral disks of the target galaxies - were excluded from the detection process. A final list of sources detected in all three filters was produced; there were 774 objects in NGC 891 and 998 in NGC 4013.

\section{2. $\quad$ Removing Extended Sources}

Objects that appear extended in our $\sim 1^{\prime \prime}$-resolution images are likely to be background galaxies rather than GCs. To remove these objects, we measured the FWHM of each source and plotted it versus the source's instrumental magnitude. Bright point sources form a tight sequence around the mean FWHM value for each image, with increasing scatter around the mean as the instrumental magnitude increases. We wrote software to select objects based on this pattern; the range of acceptable FWHM values for point sources increases with source magnitude. If a source failed the FWHM criteria for a point source of a given magnitude in any one filter, it was removed from the list of potential GC candidates. After this step, the 
number of sources remaining was 490 for NGC 891 and 488 for NGC 4013.

\subsection{Aperture Photometry}

Aperture photometry was performed on the remaining point sources in the $B V R$ images of NGC 891 and NGC 4013, using an aperture of radius equal to the mean FWHM PSF for the specific image. An aperture correction was derived for each image by measuring the mean difference in the total magnitude and the magnitude within a one-FWHM-radius aperture. The aperture corrections ranged from -0.229 to -0.308 with errors of 0.001 to 0.005 . Final, calibrated total magnitudes were calculated for the point sources in the images by applying the appropriate aperture correction and photometric calibration coefficients, as well as a Galactic extinction correction derived from the reddening maps of Schlegel, Finkbeiner, \& Davis (1998). The Galactic extinction corrections for NGC 891 were $\mathrm{A}_{B}=0.277, \mathrm{~A}_{V}=0.209$, and $\mathrm{A}_{R}=0.168$, and for NGC 4013 were $\mathrm{A}_{B}=0.073, \mathrm{~A}_{V}=0.055$, and $\mathrm{A}_{R}=0.045$.

\subsection{Final Selection of GC Candidates}

The last step in the GC candidate selection process was to choose objects with $B V R$ magnitudes and colors that match those expected for GCs at the distance of the host galaxy. We executed this step using the same basic method described in the previous survey papers (RZ01, RZ03, RZ04, and R07). GCs were assumed to have $M_{V} \sim-11$ to -4 , based on the GCLF of the Milky Way and other giant galaxies (Ashman \& Zepf 1998). Objects within this magnitude range (given the galaxy distances in Table 1) were checked to determine whether their $B V R$ colors were like those expected for GCs with metallicities between $[\mathrm{Fe} / \mathrm{H}]$ of -2.5 and 0.0. Specifically, sources with $B-V$ and $V-R$ values (and corresponding photometric errors) that placed them within $2-3-\sigma$ of the derived relation for Galactic GCs with the specified metallicity range were accepted as GC candidates.

As explained in R07, the spiral galaxies included in our wide-field survey typically have so few GC candidates (a few dozen, compared to hundreds or thousands for the elliptical galaxies) that we carefully examine each of the GC candidates as well as those that narrowly miss meeting the above criteria. We then adjust the selection criteria accordingly, so as not to exclude objects that are likely to be GCs or mistakenly include objects that are probably contaminating foreground stars or background galaxies. We consider things like where objects lie in the $B V R$ color-color plane, where they are located relative to the galaxy, and how the objects appear in archival HST images (see Section 4.2.2). 
For NGC 4013, we executed the usual selection steps as described above, accepting sources with $V$ magnitudes $\geq 19.9$ and $B V R$ colors within $3-\sigma$ of the $B-V$ vs. $V-R$ relation for Galactic GCs. We also decided to impose a faint magnitude limit of $V<24$.4, or $\sim 0.7-\sigma$ past the GCLF peak, in order to minimize the contamination from faint background galaxies that were not removed in the extended source cut. The final set of GC candidates in NGC 4013 includes 83 objects.

The GC candidate selection for NGC 891 was much more involved. This galaxy is at relatively low Galactic latitude ( -17 degrees vs. the more typical range for our targets, +40 to +70 degrees) and therefore has a higher surface density of Galactic stars than typical galaxy images from the survey. Imposing the usual luminosity threshold of $M_{V}=-11$ yielded a significant population of bright sources in the GC candidate list when, based on the shape of the Milky Way GCLF and that of other giant galaxies, only a few real GCs would be expected at those magnitudes. To account for this and to limit contamination from background galaxies, we imposed more stringent $V$ magnitude criteria on both ends of the luminosity function, choosing GC candidates in the range $20.7<V<23.6$ (extending from $\sim 1.3-\sigma$ brighter than the GCLF peak to 1- $\sigma$ fainter than the peak). We also used a $2-\sigma$ criterion for the $B V R$ color selection because analysis of $H S T$ images indicated that constraining the color cut would help eliminate contaminants from the sample, while still allowing real GCs to be selected. Finally, we explicitly excluded 13 objects that had count ratios in $H S T$ images that indicated that they were background galaxies or foreground stars. (The HST data analysis is discussed in Section 4.2.2).

Lastly, we added a few sources to the final list for NGC 891. We included four sources that were located very near the galaxy disk and had colors that put them just outside the color selection box, in the direction of the reddening vector. We added two more sources that had been excluded by the more stringent $V$ magnitude criteria, but nevertheless appeared to be real GCs based on the HST study we used to supplement the WIYN data (see Section 4.4). The final set of GC candidates for NGC 891 includes 43 objects.

Figures 3 and 4 show the results of the color selection. The point sources in each galaxy field that appeared in all three filters are marked with open squares and the final GC candidates are marked with filled circles. The 2- or $3-\sigma$-wide color selection boxes are also marked. Note that due to the $V$ magnitude criteria, not all objects that appear within the color selection boxes are GC candidates. For illustrative purposes, tracks are plotted in Figures 3 and 4 that show where galaxies of different morphological types (E/S0 through Irregular) at different redshifts would lie in the color-color plane. RZ01 details how the tracks were calculated. The distribution of sources looks markedly different in the NGC 4013 and NGC 891 fields and the galaxy tracks help explain why. The NGC 4013 images are both 
deeper (see Section 4.1) and located at higher Galactic latitude (+70 degrees) than the NGC 891 images. Consequently the figure is dominated by a large number of objects located in the lower left part of the $B V R$ color-color plane for NGC 4013; these are most likely faint, blue, low- to mid- $z$ background galaxies that are unresolved in our ground-based images. By contrast the NGC 891 field has relatively few objects in the lower left corner of the $B V R$ plane, and instead has a very well-populated, narrow sequence of objects running diagonally through the plane; these are likely Galactic foreground stars.

The color-magnitude diagrams (CMDs) of the GC candidates for each galaxy are shown in Figure 5. To make use of information from all three filters, the $V$ magnitudes vs. the $B-R$ colors of the sources are plotted. The two very red sources with $B-R \sim 1.9$ in the NGC 891 CMD are very close to that galaxy's disk, which may help explain their red colors; the brighter of those two sources, with $V \sim 19$, was added to our sample based on its appearance as a legitimate GC in an HST ACS study from the literature (see Section 4.4).

\section{Additional Analysis Steps}

\subsection{Completeness Testing and Detection Limits}

We quantified the point-source detection limits of the WIYN Minimosaic images by performing a series of completeness tests. In each test, 200 artificial sources, each with a point-spread-function that matched the best-fit PSF for the image and a total magnitude within 0.2-magnitude of a specified value, were added to a given Minimosaic image. The same detection steps used on the original images were then executed and the fraction of artificial sources that was recovered was recorded. The process was repeated at 0.2-magnitude intervals over a range of 5-6 magnitudes per filter. This yielded a measurement of completeness vs. magnitude for each filter and each galaxy field. The NGC 891 images are 50\% complete at $B=25.2, V=24.2$, and $R=24.1$. The images of NGC 4013 were taken under better seeing conditions and accordingly are somewhat deeper, with $50 \%$ detection limits at $B=25.6, V=25.2$, and $R=24.8$.

\subsection{Contamination}

A serious concern for ground-based imaging studies of extragalactic GC systems is contamination from point-source stars and compact galaxies masquerading as GCs in the candidate lists. We went through a series of analysis steps to try to both quantify and correct for the contamination from stars and galaxies in the GC candidate lists. 


\subsubsection{Model Prediction for Stellar Contamination}

We used a Galactic structure model (Mendez \& van Altena 1996, Mendez et al. 2000) to help us evaluate the level of stellar contamination that exists in the GC candidate lists. Quantities like the position of the Sun within the Galaxy and the fraction of stars in the Galaxy's structural components (disk, thick disk, and halo) are input to the model; it then predicts the surface density of stars within a given magnitude and color range and direction on the sky. Our experiments with the model indicate that the predicted stellar surface density does not strongly depend on the parameters like the Galaxy composition and the solar distance and height above the Galactic disk. The model predicts that the surface density of Galactic stars with $V$ magnitudes and $B-V$ colors in the same range as the samples of GC candidates is $0.06 \mathrm{arcmin}^{-2}$ for NGC 891 and $0.07 \mathrm{arcmin}^{-2}$ for NGC 4013. Note that the value for the NGC 891 field is fairly small, even though Figure 4 suggests a large number of stars in the field; this reflects the fact that we specifically reduced the stellar contamination in the GC candidate sample for that galaxy by limiting the sample to 20.7 $<V<23.6$. (If we instead impose the usual $V$ magnitude threshold of $M_{V}=-11$, the $V$ range becomes $18.6<V<23.6$ and the stellar contamination predicted by the model is nearly four times larger, at $0.23 \operatorname{arcmin}^{-2}$.)

\subsubsection{Examining the WIYN GC Candidates in Archival HST Images}

Next we investigated the level of contamination in the GC lists due to background galaxies. HST can resolve many compact background galaxies that appear as point sources in ground-based data, so we analyzed archival HST images of NGC 4013 and NGC 891 to determine whether any of the WIYN GC candidates were actually galaxies.

For NGC 4013, we retrieved Wide Field Planetary Camera 2 (WFPC2) images from the $H S T$ data archive 3 . We analyzed two pointings in $\mathrm{F} 450 \mathrm{~W}$ located $\sim 0.5^{\prime}$ from the galaxy center (HST program GO.8242, PI: Savage) and one pointing in F814W located $\sim 0.7^{\prime}$ from the galaxy center (HST program GO.6685, PI: Huizinga). We requested that "On-the-fly" processing be applied to the images before retrieval and we used the STSDAS task gcombine to stack multiple pointings taken in the same filter. Twenty-four of the WIYN GC candidates appear in one or more of the WFPC2 frames. Photometry was performed on these objects using apertures 0.5 - and 3-pixels in radius and the ratio of the flux within the two apertures

\footnotetext{
${ }^{3}$ Based on observations made with the NASA/ESA Hubble Space Telescope, obtained from the data archive at the Space Telescope Science Institute. STScI is operated by AURA, under NASA contract NAS $5-26555$.
} 
was computed. The ratio of counts in the larger aperture to smaller aperture should be significantly larger for extended background galaxies than it is for compact GC candidates (Kundu et al. 1999). This test indicated that none of the 24 WIYN GC candidates was a background galaxy. The 24 candidates were inspected visually to confirm the results of the count ratio test. Note that this does not necessarily mean that there is zero contamination from galaxies in the WIYN sample, just that the specific subset of candidates $(\sim 30 \%$ of the total) in the WFPC2 images does not appear to include any resolved galaxies.

For NGC 891, we downloaded WFPC2 images from the HST archive and Advanced Camera for Surveys (ACS) images from the Hubble Legacy Archive (HLA)4. The WFPC2 images we used were: one pointing in F814W at $0.2^{\prime}$ from the galaxy center ( $H S T$ program GO.9042, PI: Smartt), a pointing in F450W at $r \sim 3.5^{\prime}$ (HST program GO.8805, PI: Casertano), and a pointing at $r \sim 8^{\prime}$ observed in F450W and F606W (HST program 9676, PI: Rhoads). These WFPC2 data were processed and analyzed in the same manner as described above. Thirteen of the WIYN GC candidates were located in one or more of the WFPC2 frames, and photometry with 0.5-pixel and 3-pixel apertures indicated that one candidate was a galaxy. The ACS images we analyzed consisted of three pointings in the halo of NGC 891 that overlap the region covered by the WIYN pointing. All three pointings were taken in both the F606W and F814W filters and all were from HST program G0.9414 (PI: de Grijs). The pointings were located $2.1^{\prime}, 3.7^{\prime}$, and $6.8^{\prime}$ from the galaxy center. Images from the HLA are processed using the standard HST pipeline and combined using the Multidrizzle software. We measured the fluxes in two different apertures for 21 of the WIYN GC candidates in the ACS images and found that three of these were galaxies and ten were foreground stars. As a result of this analysis, we decided to constrain the GC candidate selection applied to the WIYN data by using a 2- $\sigma$ color selection and by explicitly excluding some of the background galaxies or stars that remained even after the $2-\sigma$ criterion was applied.

Our ability to draw general conclusions about the contamination level in the WIYN data was hampered by the small numbers of objects we could identify and measure in the $H S T$ images. Therefore, rather than using the statistics from the HST analysis (e.g., one galaxy out of $13 \mathrm{GC}$ candidates in the WFPC2 images of NGC 891) to apply some sort of global contamination correction, we opted to simply remove the extended objects from the WIYN GC candidate list and use another method to calculate an overall contamination

\footnotetext{
${ }^{4}$ Based on observations made with the NASA/ESA Hubble Space Telescope, obtained from the Hubble Legacy Archive, which is a collaboration between the Space Telescope Science Institute (STScI/NASA), the Space Telescope European Coordinating Facility (ST-ECF/ESA) and the Canadian Astronomy Data Centre (CADC/NRC/CSA).
} 
correction.

\subsubsection{Contamination Correction Based on the Asymptotic Behavior of the Radial Profile}

We constructed an initial radial profile for each galaxy's GC system by assigning the GC candidates to $1^{\prime}$-wide concentric annuli centered on the galaxy nucleus. We computed the effective area of each annulus (the total area minus the regions of the images that were excluded from the daofind search described in Section 3) and used that to calculate the surface density of GCs for the annulus. GC system radial distributions that are created from wide-field images in this manner typically exhibit a peak surface density of GCs near the center of the galaxy and then fall off monotonically with increasing radius. At some distance from the galaxy center, they then flatten out to a fairly constant surface density, as long as the images are large enough to have covered the full radial range of the GC system (e.g., Harris et al. 1985, Harris 1986, RZ01, RZ04, R07). The constant surface density in the outermost annuli can be assumed to be due to contaminating objects, i.e., point sources with magnitudes and colors like GCs that cannot be easily differentiated from real GCs in a photometric study. Thus the surface density in the outer bins can be subtracted from the overall radial distribution to correct it for contamination.

There were factors that complicated applying this type of asymptotic correction for both NGC 4013 and NGC 891. NGC 4013 has recently been shown to have a tidal stellar stream (Martinez-Delgado et al. 2009), which appears as a low-surface-brightness loop extending $\sim 6^{\prime}(\sim 26 \mathrm{kpc})$ out from and around the northeast side of the galaxy. Martinez-Delgado et al. (2009) analyzed the location of the stream relative to orbital model predictions and concluded that it was likely due to a dwarf satellite of mass $\sim 6 \times 10^{8}$ solar masses that is merging with NGC 4013. The highest-surface-brightness portion of the loop appears in our Minimosaic images on the northeast side of the disk. There may be a slightly enhanced GC population on that side of the galaxy: when one compares the location of the stream relative to the GC candidates we detect, it does appear that the candidates might be preferentially located within the stream, although the effect is very subtle and may not be real (see Section 5.2). It does seem possible that the dwarf galaxy responsible for NGC 4013's stellar stream could have GCs associated with it, based on our current picture of the best-known accreting dwarf galaxy, the Sagittarius dwarf spheroidal galaxy (Sgr dSph). Sgr dSph is being tidally disrupted by its interaction with the Milky Way and studies of its orbit and stellar populations indicate that its mass is $\sim 10^{9} \mathrm{M} \odot$ and it may have deposited as many as eight GCs into the Milky Way GC system (Ibata, Gilmore, \& Irwin 1994; Johnston et al. 1999; Layden \& Sarajedini 2000; Cohen 2004; Carraro et al. 2007; Belokurov et al. 2007; Carraro 2009). In order to 
avoid over-correcting for contamination (and thus under-counting GCs) in NGC 4013's GC system, we therefore excluded 10 GC candidates that might be associated with the stream from the asymptotic contamination correction. With these objects excluded, the radial profile of GC candidates is fairly constant in the outer two (of five) annuli and the mean surface density in these annuli is $0.38 \pm 0.09 \mathrm{arcmin}^{-2}$. (Leaving in the $10 \mathrm{GC}$ candidates leads to a mean surface density in the outer two annuli of $0.62 \pm 0.12 \mathrm{arcmin}^{-2}$. Note also that the 10 GC candidates that might coincide with the stream were removed only for the purpose of calculating an asymptotic contamination correction and were included in all subsequent analysis steps.)

NGC 891 posed a problem for other reasons. The initial radial profile created by assigning the GC candidates to 1-' bins did not have the usual shape for a GC system, but instead was relatively flat over the entire radial extent of the WIYN data. The surface density of GC candidates fluctuated between $0.41-0.96 \mathrm{arcmin}^{-2}$ in the inner five annuli (at $r \sim 1^{\prime}-5^{\prime}$ from the galaxy center), before settling down to values between $0.27-0.40 \mathrm{arcmin}-2$ in the last three annuli (at $r \sim 6^{\prime}-8^{\prime}$ ). We assumed that the last three annuli provided a reasonable estimate of the surface density of contaminating objects, so we took the asymptotic correction to be the mean of these values: $0.33 \pm 0.04 \operatorname{arcmin}^{-2}$.

The asymptotic values derived from the initial radial profiles were adopted as the final contamination corrections for NGC 4013 and NGC 891. We note that the stellar contamination levels estimated from the model (Section 4.2.1) were in both cases lower than the asymptotic contamination corrections, which is consistent with the idea that additional contamination comes from unresolved galaxies. A contamination fraction was calculated for each annulus in the radial profile by multiplying the surface density of contaminants by the effective area of the annulus and then dividing that number by the total number of GC candidates in the annulus. This radially-dependent contamination correction was later used to correct the GCLF (Section 4.3) and the final radial profile (Section 5.1).

\subsection{Determining the GCLF Coverage of the WIYN Data}

Observed GC luminosity functions were calculated for NGC 891 and NGC 4013 by assigning the $V$ magnitudes of the final GC candidates to bins 0.3 magnitude wide. A contamination correction was applied to the data by multiplying the number of GCs in a particular radial bin by the contamination fraction at that radius, as determined from the asymptotic correction. The numbers in each bin were then divided by the total completeness correction for that magnitude (the total completeness is calculated by convolving the individual completenesses in the $B V R$ filters, as described in detail in RZ01). The corrected GC 
luminosity function for each galaxy was then fitted with a Gaussian function with a peak absolute magnitude like that of the Milky Way GCLF, at $M_{V}=-7.3$ (Ashman \& Zepf 1998), corresponding to an apparent magnitude of $V=22.3$ and 23.6 for NGC 891 and NGC 4013, respectively. We varied the dispersion of the Gaussian between 1.2 and 1.4 mag and found that the mean fraction of the theoretical GCLF covered by the observed luminosity function was $0.69 \pm 0.01$ for NGC 891 and $0.656 \pm 0.002$ for NGC 4013. Changing the bin size of the LF data, or excluding one bin from the fit, changed the mean fractional coverage by $\sim 2-6 \%$; we included this uncertainty in the error calculation in Section 5.3 .

\subsection{Combining HST Results from the Literature with the WIYN Results}

The GC systems of the two galaxies we observed with WIYN have been studied using HST data: Goudfrooij et al. (2003; hereafter G03) analyzed NGC 4013 and Harris et al. (2009; hereafter H09) analyzed NGC 891. The HST studies are complementary to our analysis: they have smaller radial coverage but higher resolution than the ground-based data and allow a search for GCs further into the galaxy disks. We used the results from these studies in combination with the WIYN data to produce a radial distribution for each galaxy's GC system. We include the HST results in all subsequent analysis steps.

G03 analyzed HST WFPC2 images of NGC 4013 and six other spiral galaxies. The images of NGC 4013 were taken in the F555W and F814W filters and cover the central $\sim 1.5-2^{\prime}$ around the galaxy. The WFPC2 field-of-view is plotted in Figure 2, along with the location of the WIYN pointing. G03 used steps similar to those we executed on the WIYN images to detect and select point-source GC candidates by their $V$ magnitudes and $V-I$ colors. They published a list of the 50 brightest GC candidates in NGC 4013; these have $V$ $=21.8$ to 24.4. The $V=24.4$ faint limit matches the limit we imposed on the WIYN data. We compared each object in the G03 list to the WIYN GC candidate list, to examine in detail the differences in GC selection in the two data sets. Twenty-five of the 50 objects in the G03 list are located in the galaxy disk, in areas of the WIYN images that were masked

out because we could not reliably detect GCs there. Another 20 of the $50 \mathrm{GC}$ candidates appear in both the WIYN list and the G03 list. Of the five remaining from the G03 list, three barely appear in our WIYN images and fall below our source detection limits. Two others appear in the WIYN images but were eliminated as GC candidates because they had large FWHM values or $B V R$ colors outside the expected region. We retained all 50 GC candidates in the G03 list to use in the subsequent analysis steps.

We assumed that the contamination level in the HST candidate list was negligible; G03 used detection software that characterized the sharpness and roundness of a source to 
remove extended objects, and for magnitudes brighter than $V \sim 24$ the contamination from unresolved background galaxies in these types of HST images should be very low (Kundu et al. 1999). To estimate the GCLF coverage of the HST data, we plotted the completenesscorrected luminosity function of the GC candidates and fitted it with Gaussians of varying dispersions, in the same manner as for the WIYN sample. The mean fractional coverage (averaged for three different Gaussian dispersions) is $0.75 \pm 0.01$. The completeness curves were taken from G03; the completeness correction was minimal, because the HST data are $\sim 90-100 \%$ complete to $V=24.4$.

H09 used deep HST ACS data to identify GC candidates in three fields (which they designated H1, H2, H3) covering part of the disk and inner halo of NGC 891. The locations of the H09 ACS fields, relative to the WIYN pointing, are shown in Figure 1, Each field was imaged in the F606W and F814W filters and H09 selected a sample of possible GC candidates via visual inspection. They refined the sample based on the size, shape, ellipticity, magnitude, and color of each candidate; their final published list of objects consists of 16 "Best" GC candidates and 27 more that may be GCs or contaminants. To simplify the process of combining the HST and WIYN results, we opted to include in our analysis only the sources in the H1 and H2 fields from H09. The H3 field covered an area beyond the boundaries of the WIYN images and H09 identified only five candidates in the field: two "best" GC candidates and three they tentatively label as galaxies; we did nothing more with the H3 pointing or sources.

We examined each object in the H09 list and created a final list of GC candidates from the H09 study to include in the combined ( $H S T$ plus WIYN) radial profile. The final HST GC candidate list has 21 objects and includes the "Best" candidates from H09, plus several objects that were marked "Bulge" or "Disc" by H09 (to denote that they might be massive open clusters in the Bulge or Disk of NGC 891). None of the objects that were noted as possible contaminants by H09 (e.g., designated "Star?", "Galaxy?", or "LSB") survived the source-by-source review process; in some cases, the objects were clearly galaxies, even in the ground-based WIYN images. As part of this same process, we also compared the lists of GC candidates from H09 and the WIYN data and investigated the differences between them. There were very few overlaps between the WIYN and HST GC candidate lists. Fifteen of the 21 H09 GC candidates were either in regions of the WIYN images that had been masked out (regions in the spiral disk where point sources could not be reliably detected) or located off the edge of the WIYN images. Four objects were already identified as WIYN GC candidates. Another object had been excluded from the WIYN GC candidate list because it was bright $(\mathrm{V}=19.3)$; we decided to add it back to the WIYN list since it was so clearly a GC candidate in the HST data. Finally, one object had been excluded from the WIYN GC candidate list because it is extended in the WIYN images. 
The contamination level in the final sample of $21 \mathrm{GC}$ candidates from H09 should be minimal; NGC 891 is only $\sim 8 \mathrm{Mpc}$ away so GCs are sometimes resolved in ACS images and can usually be distinguished from point-source foreground stars. H09 make use of this and shape information to remove stars and galaxies from the sample. The H09 data are very deep, reaching 50\% completeness at $V \sim 29$ and $I \sim 27-28$, whereas the theoretical GCLF for this galaxy should peak at $V \sim 22.3$ and go to zero at $V \sim 26$. Because of this, we assumed that the GCLF was fully sampled by the H09 data and that any GCLF coverage correction would be zero or negligible.

\section{Results}

\subsection{Radial Distributions of the GC Systems}

The final, corrected radial distributions of each galaxy's GC system were produced first by assigning the GC candidates from WIYN and the HST studies of G03 or H09 to one of a series of concentric annuli, according to their projected radial distances from the galaxy center. For the WIYN data, we used annuli of width $1^{\prime}$ (as explained in Section 4.2.3). The HST GC candidates were more densely packed and located within a smaller region, so were binned more finely, into annuli of width $0.5^{\prime}$. The inner boundary of the first annulus was always placed just inside the radial position of the innermost GC candidate. An effective area, that excludes missing or masked regions, was calculated for each annulus. The number of GCs in each annulus was corrected for contamination and GCLF coverage when appropriate, using the corrections discussed in previous sections. We then divided the corrected number of GCs by the appropriate effective area to yield the surface density of GCs in each annulus. The error on the surface density includes Poisson errors on the number of GCs and the number of contaminating objects. The final radial distributions for the GC systems of NGC 891 and NGC 4013 are listed in Tables 3 and 4 and plotted in Figures 6 and 7. The radii listed in the tables are the average projected radii of the unmasked pixels in the corresponding annulus. For each projected radius, the tables list the surface density and error, the fraction of the annulus that was observed (excluding missing or masked regions), and the source of the measurement (whether it came from the WIYN or HST data).

We note here that neither of the published HST studies of these galaxies included a calculation of the GC system radial profile. G03 stated that low number statistics and

missing spatial coverage made it too difficult to evaluate the radial distribution of GCs, and they estimated the total number of GCs instead by comparing the number of GCs detected at various spatial locations around NGC 4013 with the number that would be detected at that same location in the Milky Way. They note that this type of analysis implicitly assumes 
that the spatial distribution of GCs in the target galaxy is similar to that of the Milky Way. H09 comment on the "severely limited area coverage" of the ACS study and similarly choose to analyze the spatial distribution and population of NGC 891's GC system by comparing the number of GC candidates in their fields with the number that would fall within the same projected area around the Milky Way. By combining the data from HST that covers the inner few arc minutes of the galaxy with the ground-based WIYN images that extend to larger radii, we are able to quantify the radial profile of the GC systems and empirically determine how far the systems extend from the host galaxies. This leads to better-determined values of the total number and specific frequency of GCs for each system (see the next section).

The radial distribution of the NGC 4013 GC system (Figure 7) has the well-behaved, regular appearance typical of the GC systems of most of the other spiral galaxies in our survey (see RZ03 and R07). The surface density of GCs is at its highest value near the galaxy center and then decreases monotonically until it eventually reaches zero within the errors, in this case in the $r=2.8^{\prime}(\sim 12 \mathrm{kpc})$ annulus. Conversely, the profile of NGC 891's GC system is not well-behaved. The combined HST plus WIYN profile (Figure 6) shows positive surface density in the first two annuli (from the HST data) inside $1^{\prime}$, but then the surface density plunges in the third annulus at $1.25^{\prime}$, then fluctuates between zero and positive surface density at $r \sim 1.5^{\prime}-3.4^{\prime}$. All the annuli beyond $r \sim 3.4^{\prime}(\sim 8 \mathrm{kpc})$ have zero surface density within the errors. Without the H09 GC candidates to add to the WIYN sample, we would likely have concluded that NGC 891's GC system was not definitively detected in the WIYN images. (The BVR color-color plot in Figure 4 and the color-magnitude diagram in Figure 5 likewise indicate, at best, a weak WIYN detection of the GC system: there is little or no overdensity of objects within the GC selection box in the color-color diagram, and the objects in the color-magnitude diagram are not tightly clustered in the same region of the $V-(B-R)$ plane, as they should be.) Likewise, with only the HST data we would not have known how far out to integrate the GC system profile in Section 5.3 ,

The corrected radial distributions were fitted with de Vaucouleurs profiles (of the form $\log \sigma_{\mathrm{GC}}=a 0+a 1 r^{1 / 4}$ ) and power law profiles (of the form $\log \sigma_{\mathrm{GC}}=a 0+a 1 \log r$ ). For NGC 891, the power law had a slightly smaller $\chi^{2}$ value (0.9 vs. 1.1 for the de Vaucouleurs law); for NGC 4013, the opposite was true, and the de Vaucouleurs $\chi^{2}$ was smaller (0.4 vs. 0.5 for the power law). The best-fit coefficients for both the de Vaucouleurs law and power law, for both galaxies' GC systems, are given in Table 5. The function with the smallest $\chi^{2}$ is plotted in the bottom panels of Figures 6 and 7 and used to calculate the total number of GCs in Section 5.3 ,

One of the quantities we evaluate in the wide-field survey is the radial extent of each galaxy's GC system, which we define as the point in the radial profile at which the surface 
density of GCs becomes consistent with zero and remains zero (within the errors) to the outer limit of the data (R07). We tabulated this measure of radial extent for nine survey galaxies in R07 and we can now add two more points to the sample. The radial extent for NGC 891 is $9 \pm 3 \mathrm{kpc}$ and for NGC 4013 is $14 \pm 5 \mathrm{kpc}$; the errors include distance errors and uncertainties associated with determining the extent from binned radial profile data. The galaxy stellar masses, derived by combining $M_{V}^{T}$ (Table 1) with mass-to-light ratios from Zepf \& Ashman (1993), are $\log \left(M / M_{\odot}\right)=11.0$ and 10.9 for NGC 891 and NGC 4013, respectively. In R07, we showed that the more massive galaxies in the survey generally have more extended GC systems and we derived a quantitative relationship between the GC system radial extent and the host galaxy mass. As in R07, we again fit a line and a second-order polynomial to the data. The best-fit coefficients of both the line and the curve are consistent within the errors with the corresponding coefficients derived in R07. The data and new best-fit second-order curve are plotted in Figure 8 . The curve has the form

$$
y=\left((49.9 \pm 9.2) x^{2}\right)-((1080 \pm 209) x)+(5860 \pm 1190),
$$

where $x$ is $\log (M / M \odot)$ and $y$ is the radial extent in kiloparsecs. This relationship can be used to determine in advance how much spatial coverage is needed to image the GC system of a given galaxy.

\subsection{GCs and the Stellar Stream of NGC 4013}

As mentioned in Section 4.2.3, NGC 4013 was found by Martinez-Delgado et al. (2009) to be surrounded by a low-surface-brightness stellar stream, possibly due to a dwarf galaxy merging into the larger galaxy's halo. In the process of calculating the asymptotic contamination correction for the GC system of NGC 4013, we identified 10 GC candidates that coincide spatially with the stellar stream and therefore might have originated in the dwarf galaxy that is being tidally disrupted. Without velocity measurements for these objects (and perhaps even then), we cannot definitively say whether or not the GC candidates are actually moving with the stream material. Since only three of the relevant GC candidates have $V \leq 22.0$ and the other seven have $23.4<V<24.3$, velocity measurements would be difficult to obtain for the majority of the 10 candidates, given the limits of today's spectroscopic facilities.

We explored various methods for investigating, based on the WIYN images, whether there are indeed "stream" GCs in NGC 4013. One straightforward approach is to see whether there is an overdensity of GC candidates in the region of the stream loop on the galaxy's 
northeast side relative to other areas of the GC system. To do this, we defined a rectangular region on the WIYN images of NGC 4013 that isolates the part of the tidal stream that included the $10 \mathrm{GC}$ candidates that are spatially coincident with the stream. We also defined a "control" region with the same total area and mean radial distance from the galaxy center and therefore that samples an analogous part of the galaxy's GC system. There are 13 GC candidates located within the stream region and 10 in the control region; assuming Poisson statistics, these numbers are equivalent. Therefore we cannot say, based on our imaging data and a simple number density argument, whether or not there are GCs associated with the tidal stream in NGC 4013. This is perhaps not surprising, since it has taken years of work - including theoretical modeling, accurate velocity measurements and detailed stellar population studies - to show that Sgr dSph in our own galaxy's halo is host to several GCs that were once thought to be part of the Milky Way GC system (e.g., Ibata, Gilmore, \& Irwin 1994, Layden \& Sarajedini 2000, Cohen 2004, Carraro et al. 2007).

\subsection{Total Numbers and Specific Frequencies of GCs}

\subsubsection{Total Specific Frequency}

We calculated the total number of GCs $\left(N_{G C}\right)$ in each galaxy's system by integrating the function that provides the best fit to the final radial distribution. For NGC 891, we integrated the best-fitting power law given in Table 5. The inner radius of the integration was taken to be $0.2^{\prime}$, the inner boundary of the first annulus of the observed radial profile. The outer radius of the integration was $3.7^{\prime}$, because beyond that radius the surface density of GCs is consistent with zero (within the errors) in the rest of the annuli. We had to make assumptions about the behavior of the radial profile near the galaxy center, in regions we could not observe. For NGC 891, this was the central $0.2^{\prime}$ of the GC system, or the central $\sim 490$ pc for $m-M=29.61$. We calculated the number of GCs that had been missed in this inner region given two possibilities: (1) that the profile inside the unobserved region continued to follow the best-fit power law to small $r$, or (2) that the profile flattened in the inner region (i.e., the GC surface density in the unobserved region equaled the value in the innermost observed annulus). Adding the number of GCs in the observed portion of the system to the number given these two assumptions for the inner region yielded two estimates for $N_{G C}$ : 53 for the flat inner profile and 81 if we extended the power law to small $r$. The mean of the two estimates, $N_{G C}=70 \pm 20$, is the final value given in Table 6 .

For NGC 4013, the de Vaucouleurs law in Table 5 was the best-fitting function to the observed radial profile. We integrated the de Vaucouleurs law from $0.08^{\prime}$ (the inner boundary

of the first annulus in the observed profile) to $3.25^{\prime}$ (the outer boundary of the $2.8^{\prime}$ annulus, 
which is where the GC surface density reaches zero within the errors and remains zero for the rest of the observed profile). For the region inside $0.08^{\prime}$, we assumed a flat radial profile or a de Vaucouleurs law all the way to $r=0$. The results given these two assumptions were very close to each other: $N_{G C}=138$ for the flat inner profile and 144 if the de Vaucouleurs law was continued to $r=0$. Again we have taken the average of the two values for $N_{G C}$ to be the final value for the GC system: 140土20 (Table 6).

We also calculated luminosity- and mass-normalized specific frequencies for each galaxy's GC system. The total number of GCs normalized by the $V$-band luminosity of the host galaxy is defined as

$$
S_{N} \equiv N_{G C} 10^{+0.4\left(M_{V}+15\right)}
$$

(Harris \& van den Bergh 1981) and the number of GCs normalized by the host galaxy stellar mass is defined as

$$
T \equiv \frac{N_{G C}}{M_{G} / 10^{9} \mathrm{M}_{\odot}}
$$

(Zepf \& Ashman 1993). We used $M_{V}^{T}$ from Table 1 and the galaxy mass (designated $M_{G}$ above) from Section 5.1 to calculate $S_{N}$ and $T$; specific frequencies are given in Table 6 .

The errors on $N_{G C}$ given in Table 6 include contributions from the following sources of uncertainty: (1) the change in the calculated GCLF coverage depending on the assumed intrinsic GCLF dispersion and how the LF data were binned; (2) Poisson errors on the number of GCs and contaminating objects; and (3) uncertainties in the number of GCs in the inner, unobserved region of the system. The errors on the specific frequencies $S_{N}$ and $T$ also take into account the error on the total galaxy magnitude; we assumed that the uncertainty in the extinction-corrected galaxy magnitude was three times the error on $V^{T}$ given in RC3 (de Vaucouleurs et al. 1991). Each of these sources of uncertainty was added in quadrature to yield the final total error value given in the table.

Next we examine how our estimates of the number and specific frequency of GCs for these galaxies compare to those from past studies. In their HST WFPC2 study of the GC system of NGC 4013, G03 estimate the total number of GCs not by constructing and integrating the radial profile, but by comparing the number of GC candidates they detect in certain regions around the galaxy to the number of GCs that would be detected at analogous locations in the Milky Way. Using this method, they calculate that NGC 4013 has $N_{G C}=$ $243 \pm 51$, which is significantly larger than our estimate of $140 \pm 20$. They combine this with 
$m-M=31.35$ and $M_{V}^{T}=-20.83$ to derive $S_{N}=1.1 \pm 0.3$ and $T=2.2 \pm 0.7$. They adopt a larger distance modulus than we do for NGC 4013, so their inferred galaxy luminosity and mass are larger than ours. Our value for $N_{G C}$ is $\sim 40 \%$ lower than their value, but our $S_{N}$ and $T$ values actually agree with the estimates given in G03 because of the larger distance modulus they assume. If we instead assume the distance modulus from G03, $m-M=31.35$, and go through all of the analysis steps to derive final numbers and specific frequencies for NGC 4013 (namely, applying a new $V$ magnitude selection to the GC sample, fitting the GCLF for both the WIYN and HST data, and calculating and integrating a new radial profile), we find $N_{G C}=160 \pm 20, S_{N}=0.7 \pm 0.2$, and $T=1.4 \pm 0.3$. The end result is that, even assuming the larger distance to NGC 4013, we derive a smaller total number of GCs in the galaxy which leads to correspondingly smaller $S_{N}$ and $T$ values.

The GC system of NGC 891 has been studied by van den Bergh \& Harris (1982) and much more recently by the H09 HST study that we have combined with our WIYN results. van den Bergh \& Harris (1982) obtained photographic observations with $\sim 1^{\prime \prime}$ seeing and counted point sources around NGC 891, looking for an overdensity of point-like objects that would signal the presence of a GC population. They found "no significant population" of GCs in NGC 891 and calculated a specific frequency consistent with zero $\left(S_{N} \sim 0.04 \pm 0.08\right)$; they speculated that the weak bulge of NGC 891 may be responsible for its apparent lack of GCs. To calculate $N_{G C}$, H09 adopt a method similar to that of G03: because their HST ACS data also cover only a small portion of the GC system, they compare the number of GC candidates they find with the number of Milky Way GCs that would fall within a corresponding projected area. From this comparison they conclude that NGC 891 has between 80 and 200 GCs and that the GC system "resembles that of the Milky Way rather closely" in terms of total number and spatial distribution of GCs. By combining the H09 GC candidate list with WIYN imaging data and constructing a radial profile for the GC system, we find that NGC 891 has a fairly small total number of GCs $(70 \pm 20)$ and small specific frequency $\left(S_{N}=0.3 \pm 0.1\right.$, compared to $0.6 \pm 0.1$ for the Milky Way). Without the HST results from H09 to augment our WIYN data, we might have concluded that NGC 891 had no detectable GC system as van den Bergh \& Harris (1982) did. This galaxy's GC system is so poorly populated, and the few GCs that are present are so close to the galaxy disk, that typical ground-based capabilities and techniques are simply not adequate to produce a definitive detection. On the other hand, combining our ground-based data with the HST data allowed us to draw more definitive conclusions than H09 about how many GCs are present in NGC 891.

Figure 9 plots the number of GCs against galaxy Hubble type (top panel) and against the log of the galaxy stellar mass (bottom panel) for the spiral galaxies in our wide-field survey, plus the Milky Way and M31. Figure 10 shows $S_{N}$ versus $M_{T}^{V}$ and $T$ versus the $\log$ 
of the galaxy stellar mass for the same set of galaxies. In R07, we calculated the mean value of $N_{G C}$, and the weighted mean values of $S_{N}$ and $T$, for the spiral galaxies in our survey plus the Milky Way and M31. These were $N_{G C}=170 \pm 40, S_{N}=0.8 \pm 0.2$, and $T=1.4 \pm 0.3$. The values we calculate for NGC 4013's GC system are very much in line with these mean values, but the $N_{G C}, S_{N}$, and $T$ values for NGC 891 are comparatively low. NGC 891 is frequently described in the literature as being similar to the Milky Way because of its morphological type, luminosity, and molecular gas distribution (e.g., Sandage 1961, van der Kruit 1984, Scoville et al. 1993), but its GC system is about half as populous as the Milky Way's. One possibility we considered is whether this galaxy has very few GCs because, despite being similar to the Milky Way in appearance, it is actually much less massive than our Galaxy. As explained in Section 5.1, we calculate $\log \left(\mathrm{M} / \mathrm{M}_{\odot}\right)=11.0$ for NGC 891. The Milky Way has $M_{V}=-21.3$ and is an Sbc spiral galaxy (Harris 1991); assuming the $M / L_{V}$ values from Zepf \& Ashman (1993), this yields a mass of $\log \left(\mathrm{M} / \mathrm{M}_{\odot}\right)=11.2$. The $\mathrm{CO}+\mathrm{HI}$ rotation curve of NGC 891 is similar to that of the Milky Way, with a rotation velocity that rises steeply near the galaxy nucleus to a maximum velocity of $250 \mathrm{~km} \mathrm{~s}^{-1}$ at $\sim 3 \mathrm{kpc}$ out to $15 \mathrm{kpc}$ (Sofue 1996), suggesting this galaxy and the Milky Way probably have comparable mass distributions. In any case, the new mean value for $N_{G C}$ for spiral galaxies in Figure 9 is $150 \pm 30$. The weighted mean values for $S_{N}$ and $T$ for the galaxies in Figure 10 are $0.5 \pm 0.1$ and $1.1 \pm 0.2$, respectively. These updated values agree within the errors with results from Chandar et al. (2004), who studied five spiral galaxies and found a mean $S_{N}$ of $0.5 \pm 0.1$ and $T$ of $1.3 \pm 0.2$. However, our new mean values are slightly smaller than the average values from the G03 study of five Sab-Sc spiral galaxies; G03 found a mean $S_{N}$ of $0.96 \pm 0.26$ and $T$ of $2.0 \pm 0.5$.

The sample of spirals we have observed with the wide-field survey is still fairly small, not all morphological types are sampled, and we have more Sb spiral galaxies in the survey than any other type. Therefore we do not wish to over-interpret the significance of the results. However, based on this small sample, there does seem to be modest variation in the number of GCs and in the GC specific frequency for galaxies with similar properties. For example, the Sb galaxy NGC 4157 and the Sab galaxy NGC 7814 have almost the same stellar mass $\left(\log \left(M / M_{\odot}=10.9\right)\right.$, but the number of GCs $(80 \pm 20$ and $170 \pm 30$, respectively) differs by a factor of two. NGC 891, NGC 2683, NGC 4157, and NGC 4013 are all classified as Sb spiral galaxies, but the number of GCs they host ranges from 70 to $>200$. The bottom line seems to be that in the overall sample, there may be a weak trend of increasing $N_{G C}$ with increasing galaxy mass (bottom panel of Figure 9), but there is enough variation in $N_{G C}$ at each galaxy mass, magnitude, and/or Hubble type to produce scatter in the $T$ versus galaxy stellar mass and $S_{N}$ versus galaxy magnitude relationships seen in Figure 10. 


\subsubsection{Specific Frequency of Blue (Metal-Poor) GCs}

Many giant galaxies - including the two with arguably the most thoroughly studied GC systems, the Milky Way and M31 - have been shown to possess GC systems with at least two populations: a blue, metal-poor population and a redder, more metal-rich populations (Zinn 1985; Zepf \& Ashman 1993; Barmbv et al. 2000; Kundu \& Whitmore 2001; Perrett et al. 2002; Kundu \& Zepf 2007; Strader et al. 2007). Various galaxy formation models have either predicted the presence of these multiple populations of GCs (Ashman \& Zepf 1992) or sought to explain them in the context of the galaxies' assembly histories (Forbes et al. 1997; Côté et al. 1998; Beasley et al. 2002). The metal-poor population of GCs is of particular interest because its low metallicity implies that it represents the first generation of GCs created in the early stages of the host galaxies' formation histories.

In previous papers from the wide-field survey (R05, R07), we estimated the number of blue (metal-poor) GCs normalized by the galaxy mass, or $T_{\text {blue }}$ :

$$
T_{\text {blue }} \equiv \frac{\left.N_{G C} \text { (blue }\right)}{M_{G} / 10^{9} \mathrm{M}_{\odot}}
$$

Here, $N_{G C}$ (blue) is the number of blue GCs and $M_{G}$ is the stellar mass of the host galaxy, calculated as described in Section 5.1. In R05 and R07, we found that $T_{\text {blue }}$ for the spiral galaxies in the survey is significantly smaller than $T_{\text {blue }}$ for the massive cluster ellipticals. This

leads to the conclusion that simply merging the GC systems of two or more late-type galaxies as we see them today cannot account for the comparatively large blue GC populations in some giant ellipticals. We also found a rough trend of increasing $T_{\text {blue }}$ values with increasing galaxy mass.

We calculated $T_{\text {blue }}$ for NGC 891 and NGC 4013 to see how they compare to the values for the other survey galaxies. and whether they fit within this overall trend. We first construct a sample of GC candidates with magnitude completeness of at least $90 \%$ in all the filters; we refer to this sample of candidates as the "complete sample". We then determine the fraction of GC candidates in the complete sample that are bluer than some pre-determined color. The color criterion we use is based on where the usual separation between the blue and red GC populations is located in elliptical galaxies; in $B-R$ this occurs at $\sim 1.23$ (RZ01, RZ04) and in $V-I$ it occurs at $\sim 1.0$ (Kundu \& Whitmore 2001).

For NGC 891, we used the WIYN data to construct a complete sample of GC candidates and found that seven of the 26 candidates (27\%) in the complete sample had $B-R$ bluer than 1.23. Because we had so few objects in the complete sample from WIYN, we also decided to construct a complete sample of GC candidates using the HST list from H09. 
The complete sample from the H09 data included 21 GC candidates; 13 of these (63\%) had $V-I<1.0$. We multiplied each of these blue fractions times the $T$ value for the galaxy's GC system to calculate $T_{\text {blue }}$ and then took the mean of the two $T_{\text {blue }}$ values as the final value. We also assigned a generous error that incorporates a wide range of possible values for $T_{\text {blue }}$, given that working with very small samples of $\mathrm{GC}$ candidates makes the entire process of the $T_{\text {blue }}$ calculation for these spiral galaxies very uncertain. The final $T_{\text {blue }}$ value for NGC 891 is $0.3 \pm 0.2$. For NGC 4013, the complete sample from the WIYN data included 51 GC candidates; of these, $38(75 \%)$ have $B-R<1.23$. Multiplying this fraction times the calculated $T$ value for NGC 4013's GC system yields a $T_{\text {blue }}$ value of $1.4 \pm 0.3$. These values are listed, along with the other total numbers and specific frequencies, in Table 6.

In Figure 11, we present $T_{\text {blue }}$ values for 16 galaxies, plotted versus the galaxies' stellar masses. Note that this is the same $T_{\text {blue }}$ figure presented in R07, but with NGC 891 and NGC 4013 added. The figure now includes: 11 spiral, S0, and elliptical galaxies from our wide-field survey (RZ01, RZ03, RZ04, R05, R07); three early-type galaxies from other multicolor, wide-field CCD studies in the literature (Forbes et al. 2001, Gomez \& Richtler 2004, Harris et al. 2004); and the Milky Way and M31 (Zinn 1985, Ashman \& Zepf 1998, Barmby et al. 2000, Perrett et al. 2002). Circles designate giant elliptical galaxies in clusters, squares are used for early-type galaxies in the field, and triangles designate spiral galaxies in the field or in groups. $T_{\text {blue }}$ for the Milky Way and M31 are the triangles with relatively small error

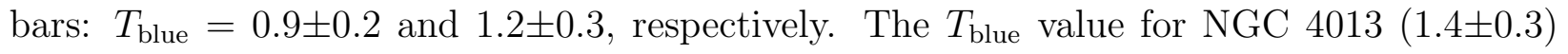
is very close to the mean derived for the other spiral galaxies without the two new points (1.2 \pm 0.1$). T_{\text {blue }}$ for NGC 891, however, is relatively low, at only $0.3 \pm 0.2$; this is comparable to the value we found for the spiral galaxy NGC 7331, 0.4 \pm 0.3 (R07).

The general trend identified in R05 - i.e., larger numbers of blue, metal-poor GCs per galaxy stellar mass for more massive galaxies - is still present in this latest version of the $T_{\text {blue }}$ figure, although (as before) there is a fair amount of scatter in the relationship. In R05 we suggested that the general trend appears consistent with a scenario in which galaxies and their GC systems are formed in a biased, hierarchical assembly process. As explained in Santos (2003), the idea is that the formation of giant galaxies began at high redshift $(z>20)$ as gaseous protogalactic building blocks collided and merged together to form larger galaxies. Merging of gaseous protogalaxies triggers the formation of GCs (in a similar way as envisioned by Ashman \& Zepf 1992). As galaxies are built up hierarchically over time, they increase both their total stellar mass and their GC population. Massive galaxies that are located in parts of the universe that have higher density (e.g., areas of the universe where galaxy clusters will eventually be located, at $z \sim 0$ ) begin this assembly process earliest, before less-massive galaxies that are located in lower-density environs. In the Santos (2003) picture, galaxy assembly and GC formation are temporarily suppressed when 
reionization occurs in the universe. The energy imparted to the intergalactic medium slows down or stops protogalactic building blocks from merging and forming stars, perhaps for a period of $\lesssim 1$ Gyr. Stars continue to evolve during this time, so that when the assembly and associated GC formation process resumes, the gas has been enriched and any GCs formed after the suppression period are metal-rich by comparison. The result of such a scenario is that today's more massive galaxies should have higher numbers of metal-poor GCs per galaxy mass than less massive galaxies, simply because they started the assembly process first; a larger percentage of their baryonic mass was in place to participate in the formation of the first generation of GCs before the temporary suppression occurred. This type of scenario also provides a natural explanation for the gap in metallicity between GC subpopulations in the Milky Way and other giant galaxies.

The curves in Figure 11] were made by G. Bryan (private communication) using a PressSchecter calculation (Press \& Schechter 1974) first described in R07. The curves show how the slope of the $T_{\text {blue }}$ trend would be expected to change as the redshift of suppression (i.e., the redshift at which galaxy assembly and GC formation are temporarily stopped) changes, for three choices of redshift: $z=7,11$, or 15. Bryan's calculation assumes that GCs form within gaseous building blocks with masses of at least $10^{8}$ solar masses and that the number of GCs in a given galaxy is proportional to the fraction of the galaxy mass that has assembled by the suppression redshift. The current data do not seem to match the $z=7$ curve, but instead suggest a larger suppression redshift of $z \sim 11-15$. (Given that Santos (2003) suggests that reionization is the mechanism that halts the formation of the first generation of GCs, it is interesting to note that the $z \sim 11-15$ curve matches well with recent estimates for the reionization redshift from WMAP (Alvarez et al. 2006)).

The biased, hierarchical scenario outlined here is not the only way that the trend in $T_{\text {blue }}$ seen in Figure 11 could arise; other possible explanations (e.g., differences in the efficiency of dynamical destruction of GCs in different types of galaxies, a trend in the relationship between galaxy luminosity and galaxy stellar mass that would affect the $\log \left(\mathrm{M} / \mathrm{M}_{\odot}\right)$ values in the figure) are discussed in R05 and R07. Our intent here is simply to show how the $T_{\text {blue }}$ and stellar mass values of NGC 891 and NGC 4013 compare with the values for the other survey galaxies. The figure - and our understanding of the relationship between $T_{\text {blue }}$ and galaxy and GC system formation and evolution — would certainly benefit from having additional well-determined $T_{\text {blue }}$ values at the high-mass end (where we only have values for two Virgo cluster ellipticals) and in the moderate-luminosity region $\left(\log \left(\mathrm{M} / \mathrm{M}_{\odot}\right) \sim 11.5-12\right)$. Accordingly, we have an ongoing effort to observe more galaxies with multi-color wide-field imaging and fill in the gap in the relevant mass range. 


\section{Summary and Conclusions}

We have combined $B V R$ imaging observations from the 9.6 ${ }^{\prime} \mathrm{x} 9.6^{\prime}$ Minimosaic imager on the WIYN 3.5-m telescope with archival and published data from HST to investigate the GC system properties of two spiral galaxies, NGC 891 and NGC 4013. The main findings of this work are as follows:

1. We used both the WIYN data and the HST data to construct radial distributions of the GC systems of the galaxies, corrected to account for missing areal coverage, the portion of the GCLF we could not observe given our detection limits, and contamination from foreground stars and background galaxies. NGC 891's GC system radial profile extends to $9 \pm 3 \mathrm{kpc}$ and NGC 4013's extends to $14 \pm 5 \mathrm{kpc}$ from the centers of the host galaxies; beyond those projected radii, the surface density of GCs is consistent with zero within the errors.

2. We fitted the radial distributions with de Vaucouleurs and power laws and integrated the best-fitting function to derive the total number of GCs. We find $N_{G C}=70 \pm 20$ for NGC 891 and $140 \pm 20$ for NGC 4013. Despite the fact that NGC 891 is often considered a Milky Way analog, we estimate that it has less than half as many GCs as the Galaxy. Its specific frequency $S_{N}$ is $0.3 \pm 0.1$ and its mass-normalized number of GCs, $T$, is $0.6 \pm 0.3$, compared to $S_{N}=0.6 \pm 0.1$ and $T=1.3 \pm 0.2$ for the Milky Way (Ashman \& Zepf 1998). We find that NGC 4013 has $N_{G C}=140 \pm 20, S_{N}=1.0 \pm 0.2$, and $T=1.9 \pm 0.5$.

3. We combine the results from this paper with results from our ongoing wide-field GC system survey and calculate the average total number and specific frequency of GCs for spiral galaxies in the survey plus the Milky Way and M31. We find $N_{G C}=150 \pm 30$, $S_{N}=0.5 \pm 0.1$, and $T=1.1 \pm 0.2$. These values are consistent with values from our most recent survey paper (R07) and from an analysis of five spiral galaxy GC systems by Chandar et al. (2004) but slightly smaller than those from a study of several spiral galaxies by Goudfrooij et al. (2003). Plots of number and specific frequency of GCs versus galaxy Hubble type, absolute magnitude, and stellar mass for our sample show no strong trends and a modest amount of scatter in GC system properties for otherwise similar host galaxies.

4. We derive the number of blue, metal-poor GCs normalized by the galaxy stellar mass ( $T_{\text {blue }}$ ) for both NGC 891 and NGC 4013 and find them generally consistent with a trend of increasing specific frequency of blue GCs with increasing galaxy mass. As was discussed in previous survey papers (e.g., R05, R07), the $T_{\text {blue }}$ trend may be a 
consequence of biased, hierarchical galaxy formation. In such a scenario, a larger fraction of the mass of high-mass galaxies is in place when the first generation of GCs is formed; the consequence is that higher-mass giant galaxies end up with larger $T_{\text {blue }}$ values than their lower-mass counterparts.

Finally, a key conclusion of this work is that, although this is not always possible, the most effective way to measure the global properties of the entire system of GCs around giant galaxies in the local universe is to combine good-quality wide-field imaging with HST observations. The ground-based images allow one to trace a GC system to its outer radial extent, while the HST data help to characterize contamination and probe further into the galaxy disk. One can then use both data sets to carefully construct a list of GC candidates and a radial profile for the system and derive more accurate global quantities that describe the GC population.

The research described in this paper was supported in part by an NSF Astronomy and Astrophysics Postdoctoral Fellowship (award AST-0302095) and by an NSF Faculty Early Career Development (CAREER) award (AST-0847109) to KLR. We are grateful to the staff at the WIYN Observatory and Kitt Peak National Observatory for their assistance during our observing runs. We also thank the anonymous referee for comments and suggestions that improved the paper. This research has made use of the NASA/IPAC Extragalactic Database (NED) which is operated by the Jet Propulsion Laboratory, California Institute of Technology, under contract with the National Aeronautics and Space Administration.

\section{REFERENCES}

Alvarez, M.A., Shapiro, P.R., Ahn, K., \& Iliev, I.T. 2006, ApJ, 644, L101

Ashman, K.M., \& Zepf, S.E. 1992, ApJ, 384, 50

Ashman, K.M., \& Zepf, S.E. 1998, Globular Cluster Systems (Cambridge: Cambridge University Press)

Barmby, P., Huchra, J.P., Brodie, J.P., Forbes, D.A., Schroder, L.L., \& Grillmair, C.J. 2000, AJ, 119, 727

Bastian, N. 2008, MNRAS, 390, 759

Beasley, M.A., Baugh, C.M., Forbes, D.A., Sharples, R.M., \& Frenk, C.S. 2002, MNRAS, 333,383 
Belokurov, V., et al. 2007, ApJ, 654, 897

Bergond, G., Zepf, S.E., Romanowsky, A.J., Sharples, R.M., \& Rhode, K.L. 2006, A\&A, 448, 155

Bridges, T.J., Rhode, K.L., Zepf, S.E., \& Freeman, K.C. 2007, ApJ, 658, 980

Brodie, J.P. \& Strader, J. 2006, ARA\&A, 44, 193

Cantiello, M., Brocato, E., \& Blakeslee, J.P. 2009, A\&A, 503, 87

Carraro, G. 2009, AJ, 137, 3809

Carraro, G., Zinn, R., \& Moni Bidin, C. 2007, A\&A, 466, 181

Chaboyer, B., Demarque, P., Kernan, P.J., \& Krauss, L.M. 1998, ApJ, 494, 96

Chandar, R., Whitmore, B., \& Lee, M.G. 2004, ApJ, 611, 220

Cohen, J.G. 2004, AJ, 127, 1545

Côté, P., Marzke, R.O., \& West, M.J. 1998, ApJ, 501, 554

de Grijs, R., O'Connell, R.W., \& Gallagher, J.S. 2001, AJ, 121, 768

de Vaucouleurs, G. \& de Vaucouleurs, A. 1964, Reference Catalogue of Bright Galaxies (Austin: University of Texas)

de Vaucouleurs, G., de Vaucouleurs, A., Corwin, H.G., Jr., Buta, R.J., Paturel, G., \& Fouque, P. 1991, Third Reference Catalogue of Bright Galaxies (New York: Springer)

Forbes, D.A., Brodie, J.P., \& Grillmair, C.J. 1997, AJ, 113, 1652

Forbes, D.A., Georgakakis, A.E., \& Brodie, J.P. 2001, MNRAS, 325, 1431

Forbes, D.A., Spitler, L.R., Harris, W.E., Bailin, J., Strader, J., Brodie, J.P., \& Larsen, S.S. 2010, MNRAS, 403, 429

Gnedin, O.Y. 2010, in Star Clusters: Galactic Building Blocks throughout Time and Space, IAU Symposium (Cambridge: Cambridge University Press)

Gomez, M. \& Richtler, T. 2004, A\&A, 415, 499

Goudfrooij, P., Strader, J., Brenneman, L., Kissler-Patig, M., Minniti, D., Huizinga, E. 2003, MNRAS, 343, 665 
Griffen, B.F., Drinkwater, M.J., Thomas, P.A., Helly, J.C., \& Pimbblet, K.A. 2009, MNRAS, 405,375

Harris, G.L.H., Harris, W.E., \& Geisler, D. 2004, AJ, 128, 723

Harris, H.C., Hesser, J.E., Bothun, G.D., Hanes, D.A., \& Harris, W.E. 1985, AJ, 90, 2495

Harris, W.E. 1986, AJ, 91, 822

Harris, W.E. 1991, ARA\&A, 29, 543

Harris, W.E. 1996, AJ, 112, 1487

Harris, W.E., Mouhcine, M., Rejkuba, M., \& Ibata, R. 2009, MNRAS, 395, 436

Harris, W.E., Spitler, L.R., Forbes, D.A., \& Bailin, J. 2010, MNRAS, 401, 1965

Harris, W.E. \& van den Bergh, S. 1981, AJ, 86, 1627

Ibata, R.A., Gilmore, G., \& Irwin, M.J. 1994, Nature, 370, 194

Johnston, K.V., Majewski, S.R., Siegel, M.H., Reid, I.N., \& Kunkel, W.E. 1999, AJ, 118, 1719

Kundu, A. \& Whitmore, B.C. 2001, AJ, 121, 2950

Kundu, A., Whitmore, B.C., Sparks, W.B., \& Macchetto, F.D. 1999, ApJ, 513, 733

Kundu, A. \& Zepf, S.E. 2007, ApJ, 660, L109

Landolt, A.U. 1992, AJ, 104, 340

Layden, A.C. \& Sarajedini, A. 2000, AJ, 119, 1760

Martinez-Delgado, D., Pohlen, M., Gabany, R.J., Majewski, S.R., Peñarrubia, J., \& Palma, C. 2009, ApJ, 692, 955

Mendez, R.A., Platais, I., Girard, T.M., Kozhurina-Platais, V., \& van Altena, W.F. 2000, AJ, 119, 813

Mendez, R.A. \& van Altena, W.F. 1996, AJ, 112, 655

Peng, E.W., et al. 2008, ApJ, 681, 197

Perrett, K.M., et al. 2002, AJ, 123, 2490 
Press, W.H. \& Schechter, P. 1974, ApJ, 187, 425

Rhode, K.L. \& Zepf, S.E. 2001, AJ, 121, 210

Rhode, K.L. \& Zepf, S.E. 2003, AJ, 126, 2307

Rhode, K.L. \& Zepf, S.E. 2004, AJ, 127, 302

Rhode, K.L., Zepf, S.E., Kundu, A., \& Larner, A.N. 2007, AJ, 134, 1403

Rhode, K.L., Zepf, S.E., \& Santos, M.R. 2005, ApJ, 630, L21

Sandage, A. 1961, The Hubble Atlas of Galaxies (Washington, D.C.: Carnegie Inst. of Washington)

Santos, M.R. 2003, in Extragalactic Globular Cluster Systems, ed. M. Kissler-Patig (New York: Springer)

Schlegel, D.J., Finkbeiner, D.P., \& Davis, M. 1998, ApJ, 500, 525

Scoville, N.Z., Thakkar, D., Carlstrom, J.E., \& Sargent, A.I. 1993, ApJ, 404, 59

Sofue, Y. 1996, ApJ, 458, 120

Spitler, L.R., et al. 2006, AJ, 132, 1593

Strader, J., Beasley, M., \& Brodie, J.P. 2007, AJ, 133, 2015

Tonry, J.L., et al. 2001, ApJ, 546, 681

Tully, R.B. 1988, Nearby Galaxies Catalog (Cambridge: Cambridge University Press)

van den Bergh, S. \& Harris, W.E. 1982, AJ, 87, 494

van der Kruit, P.C. 1984, A\&A, 140, 470

Verheijen M.A.W. \& Sancisi R., 2001, A\&A, 370, 765

West, M.J., Côté, P., Marzke, R.O., \& Jordán, A. 2004, Nature, 427, 31

Whitmore, B.C. \& Schweizer, F. 1995, AJ, 109, 960

Zepf, S.E., \& Ashman, K.M. 1993, MNRAS, 264, 611

Zinn, R. 1985, ApJ, 293, 424 


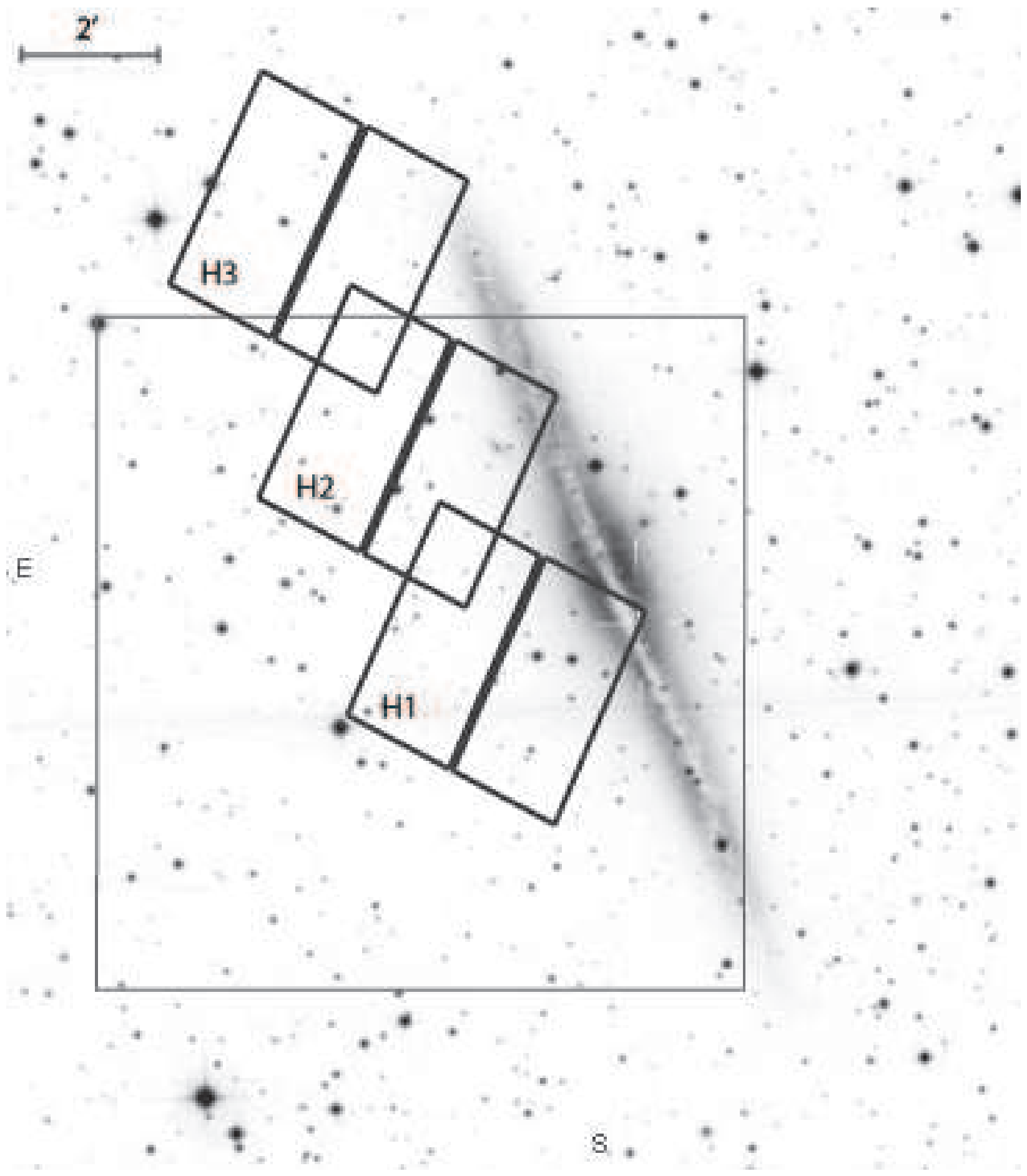

Fig. 1.- Digitized Sky Survey image of NGC 891 with the location of the WIYN pointing (large box) superimposed. The three smaller boxes marked H1, H2, and H3 show the locations of the HST ACS fields analyzed by Harris et al. (2009) and discussed in Section 4.4 . 


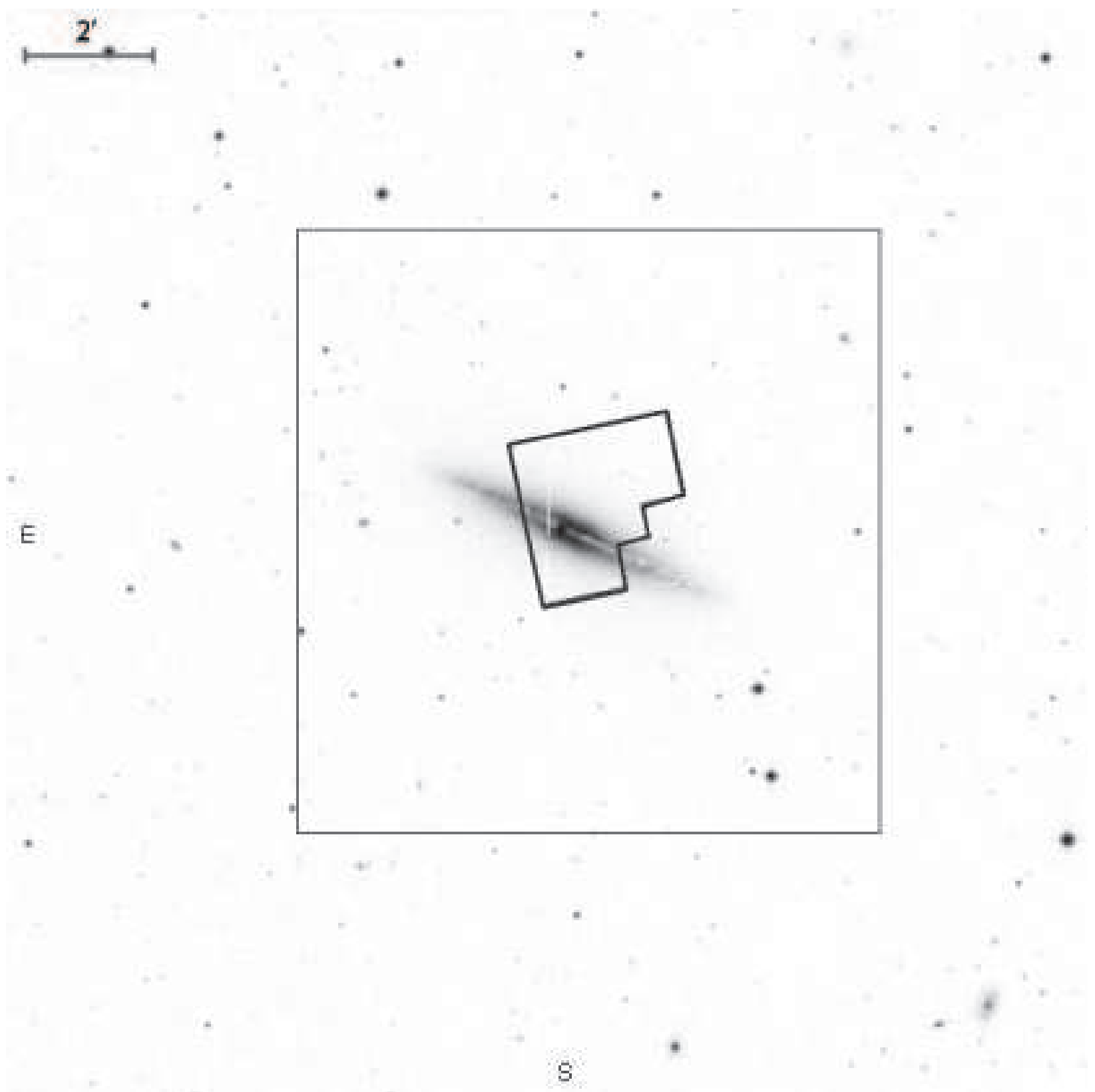

Fig. 2.- Digitized Sky Survey image of NGC 4013 with the location of the WIYN pointing (large box) superimposed. The HST WFPC2 pointing analyzed by Goudfrooij et al. (2003) and discussed in Section 4.4 is also marked. 


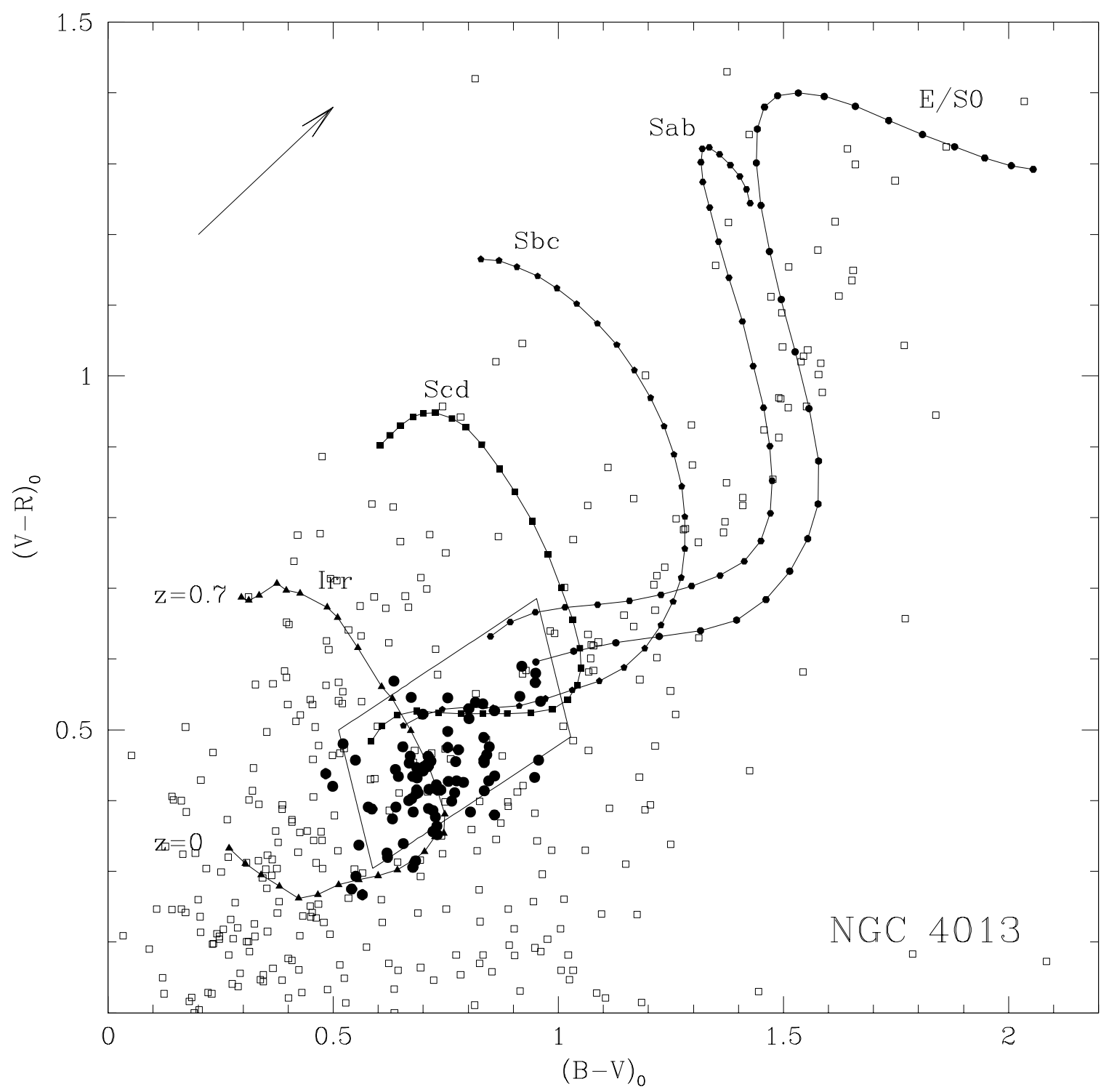

Fig. 3.- Color selection of GC candidates in NGC 4013. Open squares are the 488 point sources detected in all three filters; filled circles are the final set of $83 \mathrm{GC}$ candidates. Because $V$ magnitude criteria were imposed, not all objects within the color selection box are GC candidates. A reddening vector of length $A_{V}=1 \mathrm{mag}$ appears in the upper left-hand corner. The box shows the boundaries of the color selection criteria. The curves show where galaxies with different morphological types, located at redshifts from $z=0$ to 0.7 , would lie in this color-color plane. 


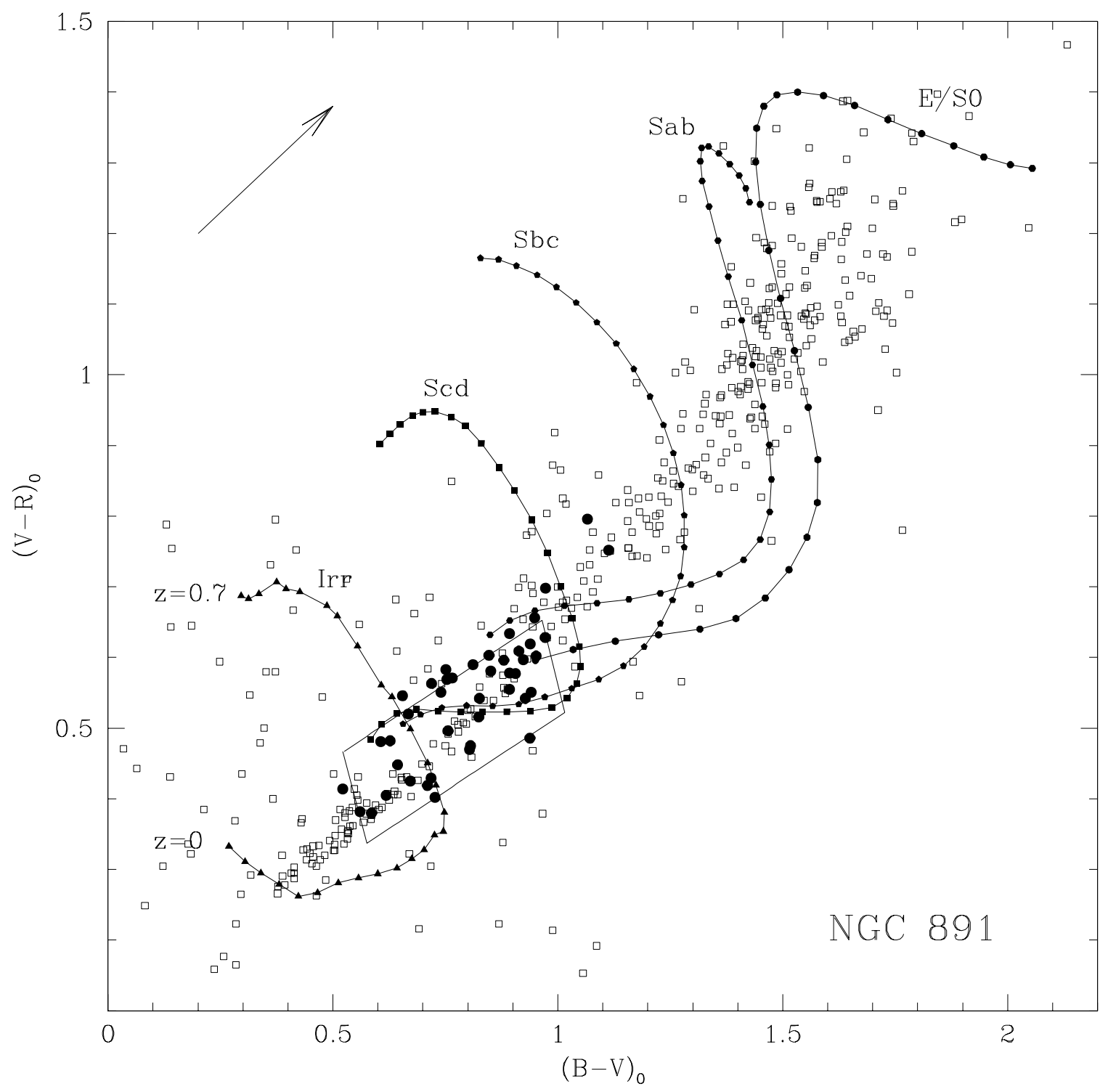

Fig. 4.- Color selection of GC candidates in NGC 891. Open squares are the 490 point sources detected in all three filters; filled circles are the final set of 43 GC candidates. Because $V$ magnitude criteria were imposed, not all objects within the color selection box are GC candidates. The other features of the plot (reddening vector, galaxy curves) are as in Figure 3 , 

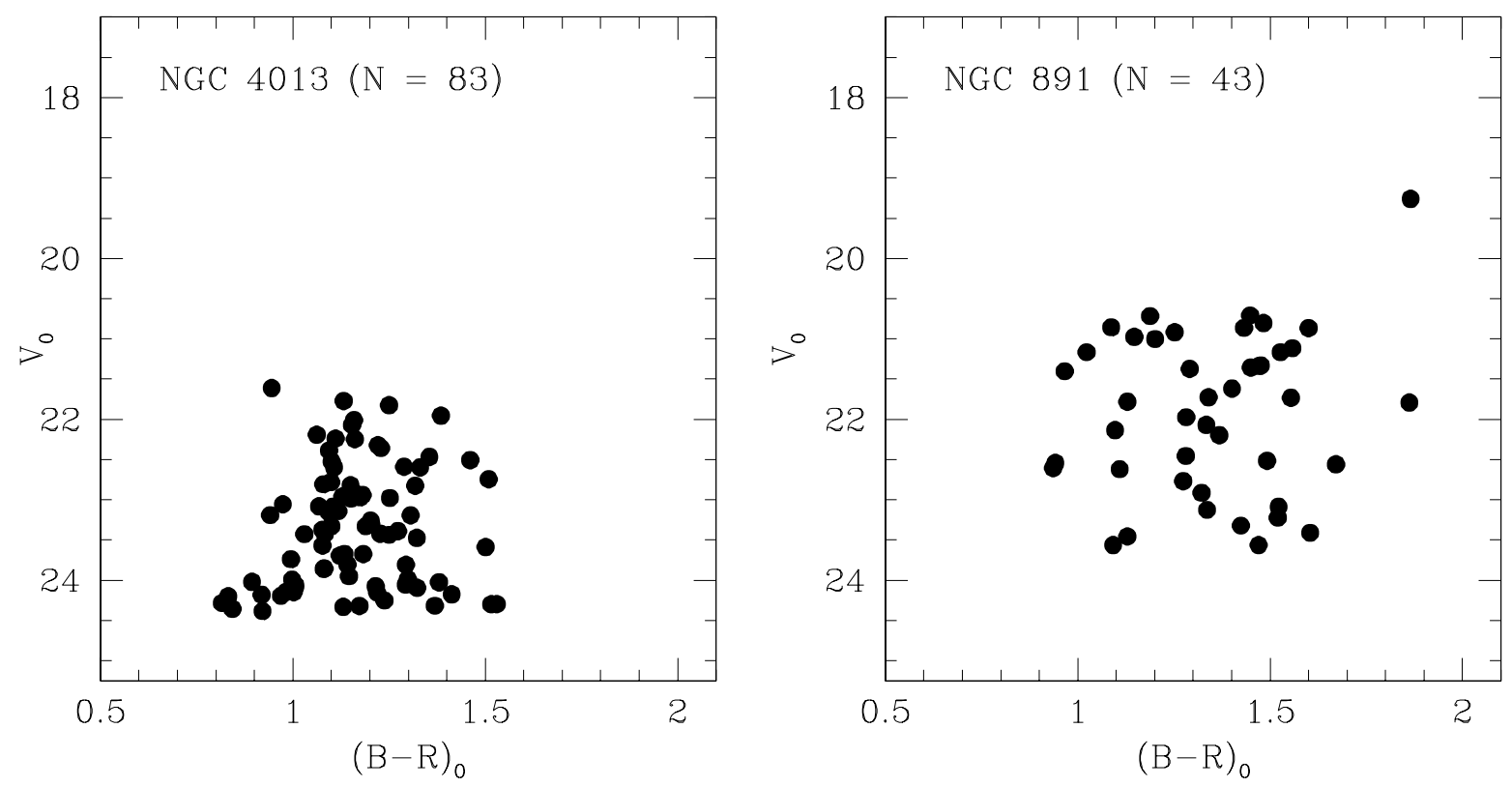

Fig. 5.- $V$ magnitude versus $B-R$ color for the final set of GC candidates in NGC 891 and NGC 4013. The magnitudes and colors have been corrected for Galactic extinction in the direction of the galaxy fields, but not for absorption internal to the galaxies themselves. 

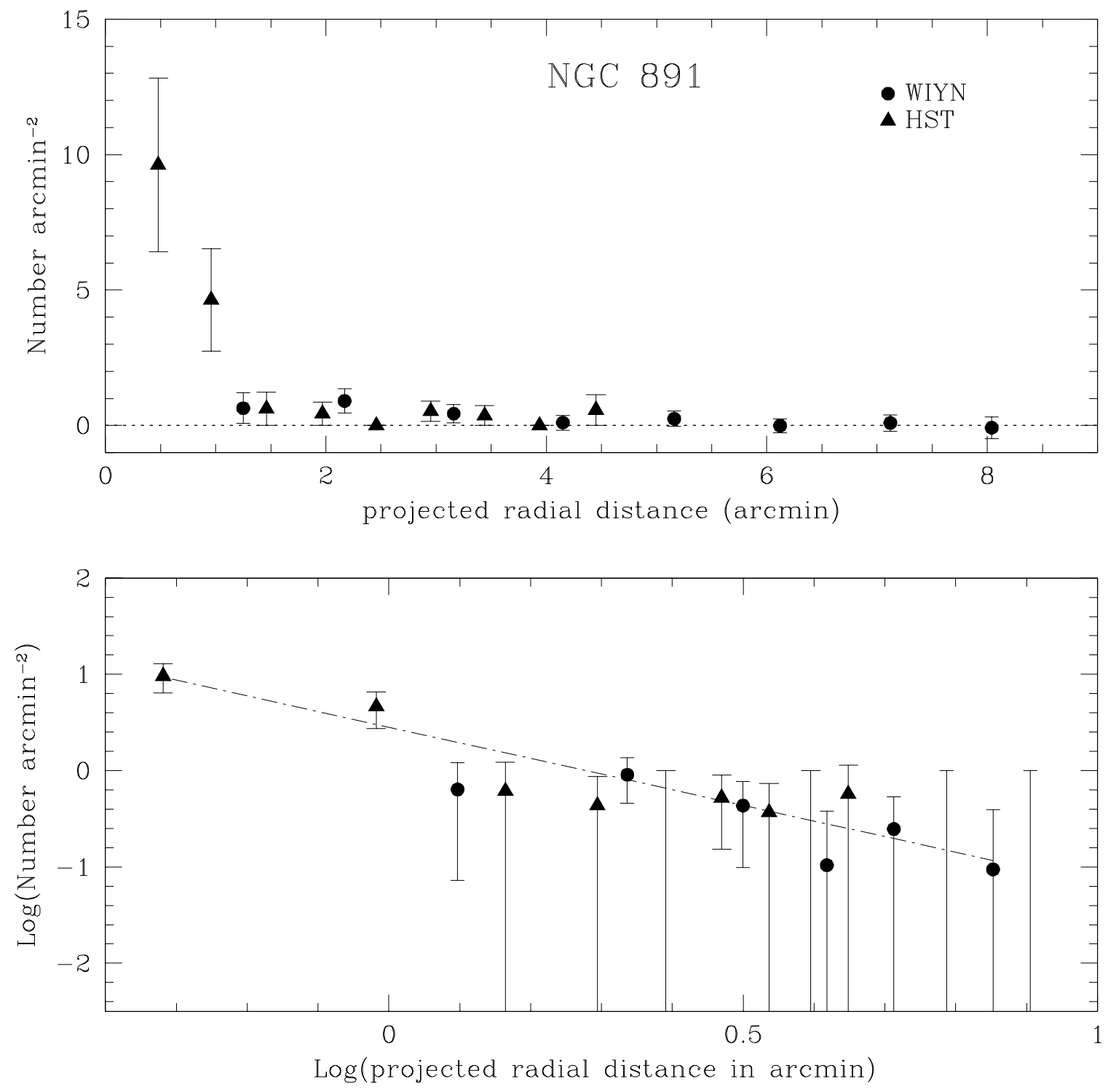

Fig. 6.- Radial distribution of GCs in NGC 891, plotted as surface density vs. projected radial distance, $r$ (top), and as the log of the surface density vs. $\log$ of $r$ (bottom). The horizontal dotted line in the top panel marks zero surface density. The dashed line in the bottom panel is the best-fit power law. The data have been corrected for contamination, areal coverage, and magnitude incompleteness, as described in Section 5.1. 

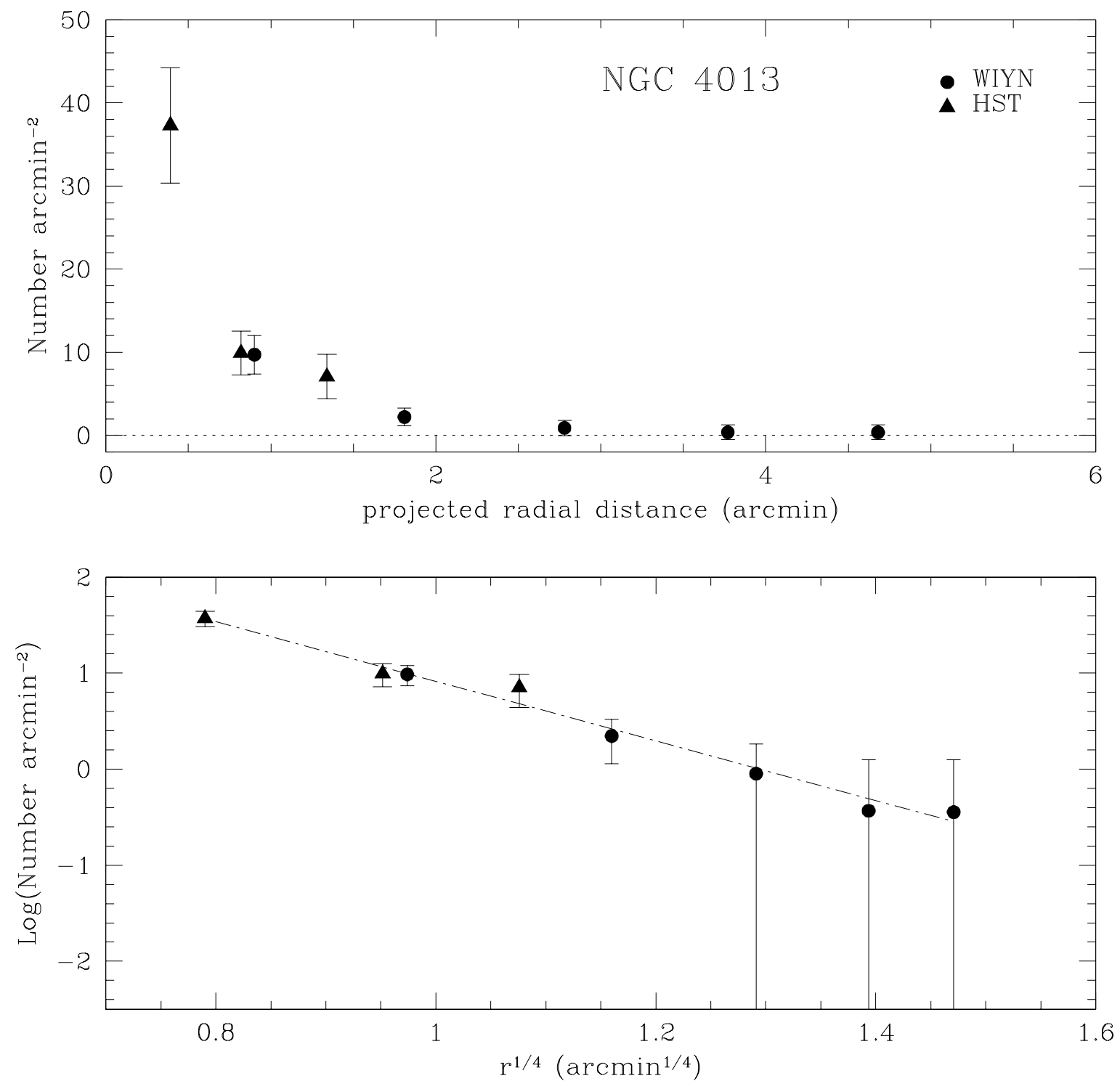

Fig. 7.- Radial distribution of GCs in NGC 4013, plotted in the same way as in Figure 6, The dashed line in the bottom panel is the best-fit de Vaucouleurs law. 


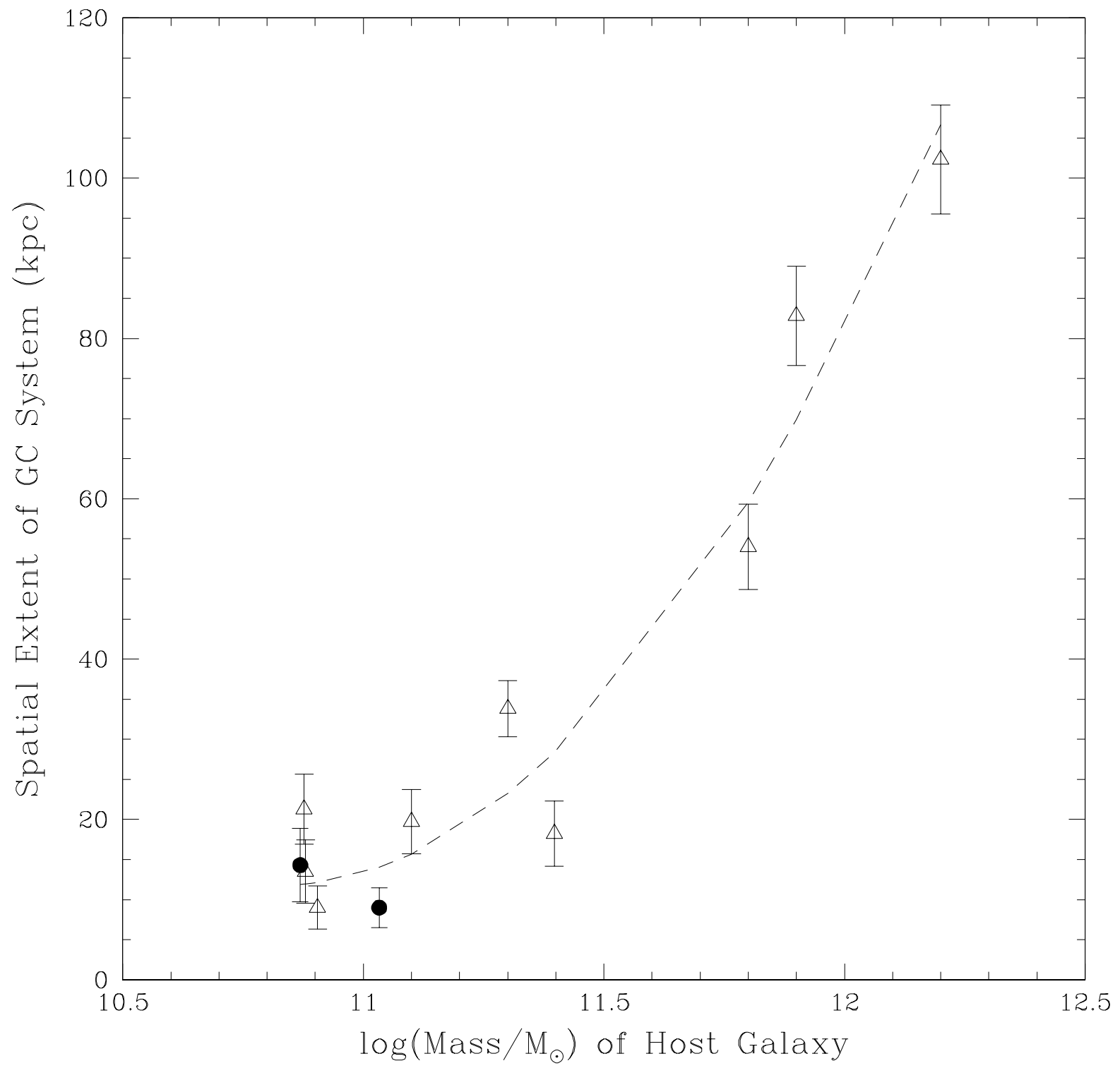

Fig. 8. - Radial extent of the GC system in kiloparsecs versus the log of the galaxy stellar mass in solar masses for 11 elliptical, S0, and spiral galaxies included in our wide-field GC system survey to date. Open triangles are the points from R07; filled circles are two new points from the current paper. The best-fit second-order polynomial (which is the same, within the errors, as the one given in R07) is shown as a dashed line. 

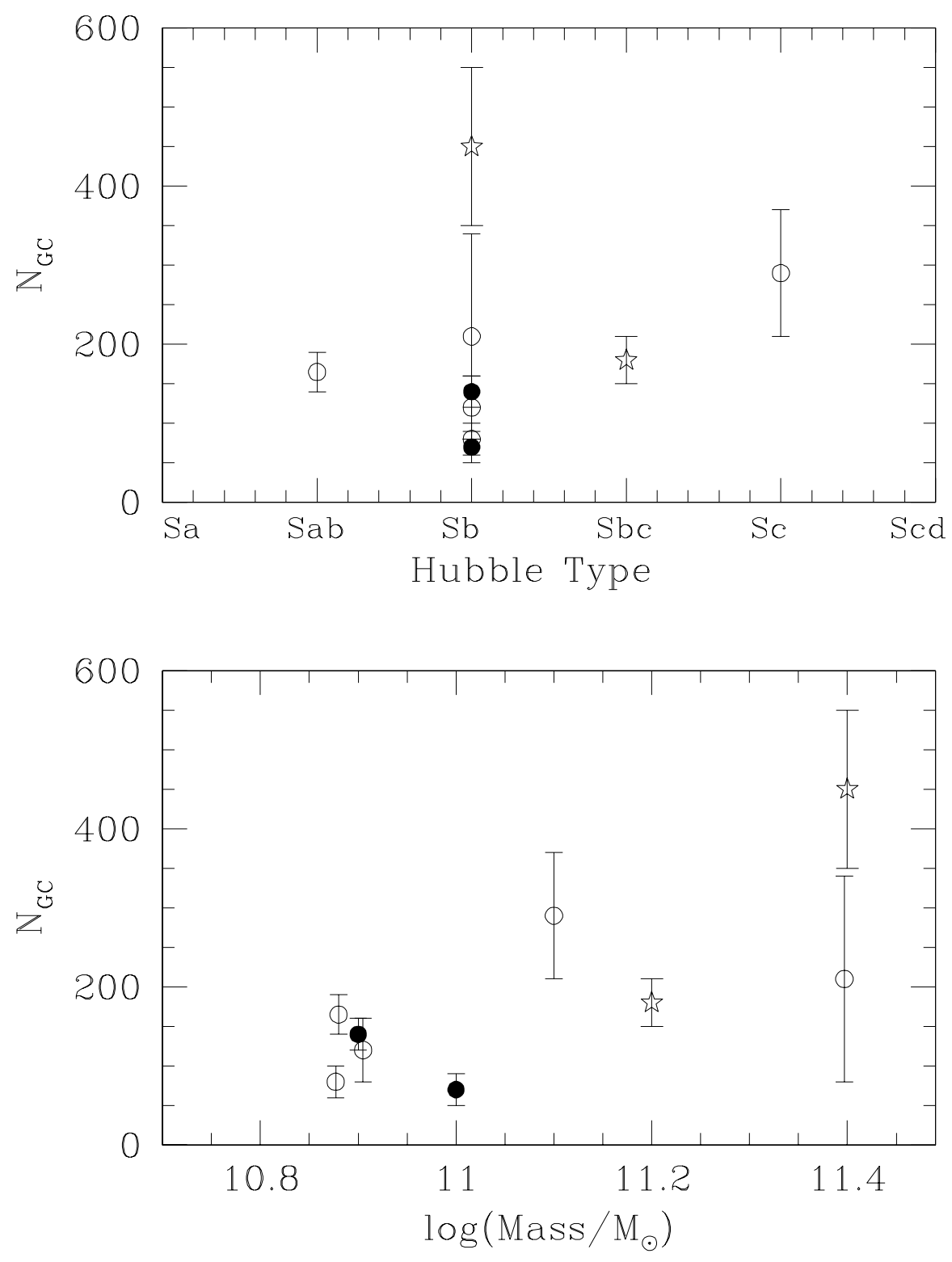

Fig. 9.- Number of GCs plotted vs. Hubble type (top) and galaxy stellar mass (bottom). Values for the spiral galaxies analyzed for our wide-field survey are plotted with filled circles (the two galaxies from the current paper) or open circles (four galaxies from R07 and one from RZ03). Values for the Milky Way (Ashman \& Zepf 1998) and M31 (Ashman \& Zepf 1998, Barmby et al. 2000; larger error bars) are plotted with open stars. 

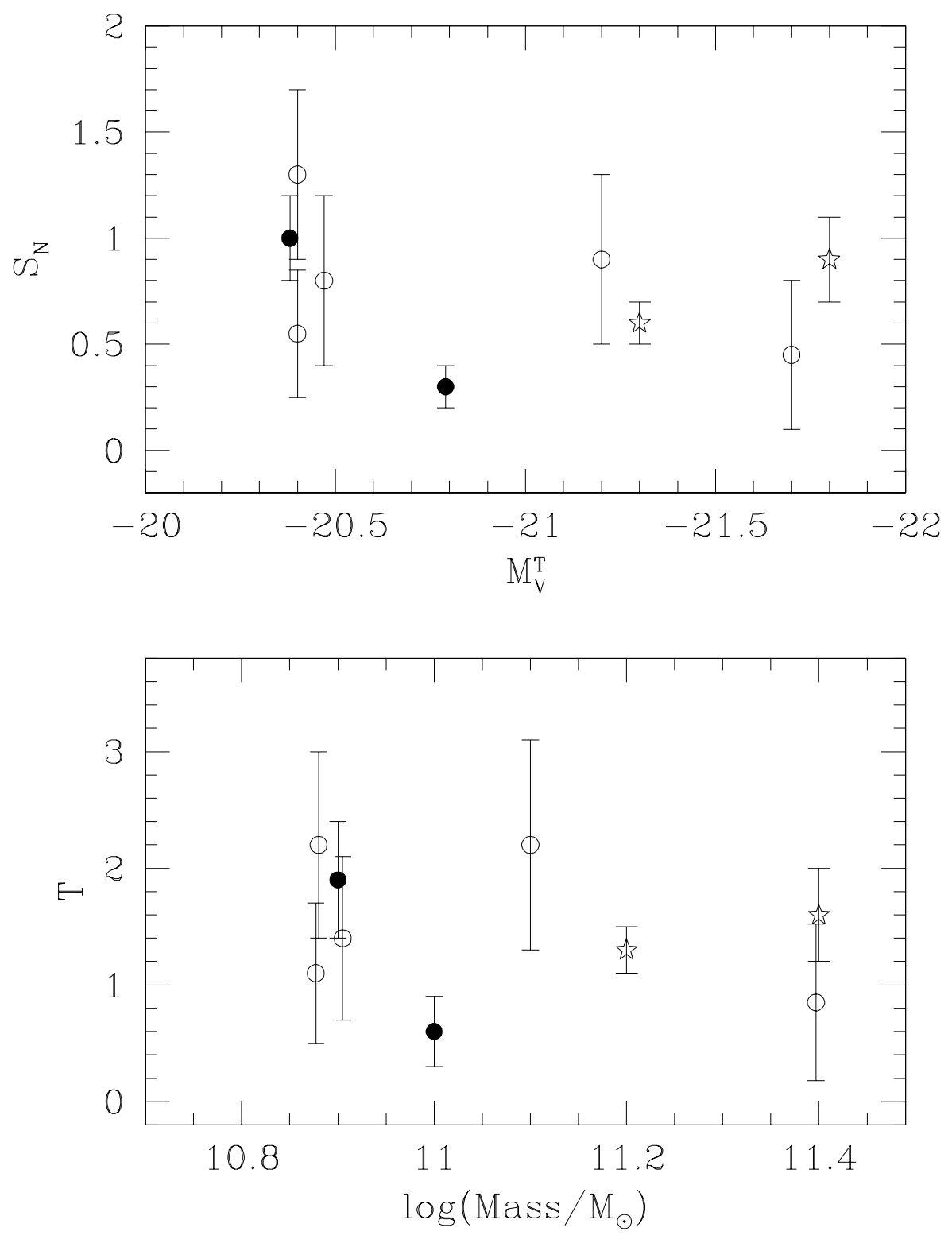

Fig. 10.- $S_{N}$ vs. $V$-band galaxy luminosity (top) and $T$ vs. galaxy stellar mass (bottom). Symbols are the same as in Figure 9. 


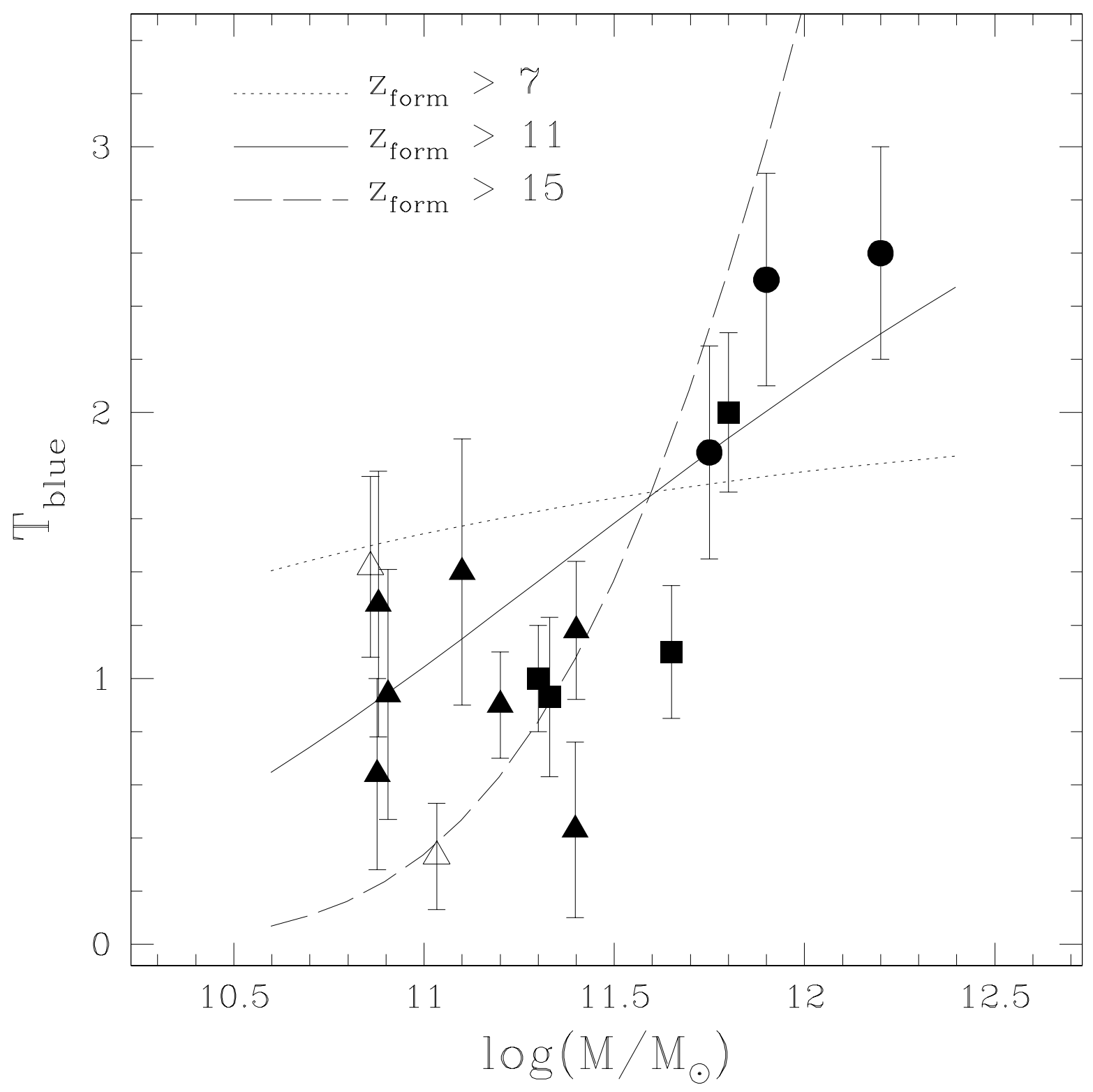

Fig. 11.- Galaxy-mass-normalized specific frequency of blue (metal-poor) GCs plotted versus the log of the galaxy stellar mass for sixteen galaxies from our wide-field survey and from other multi-color wide-field CCD imaging studies in the literature. Circles represent early-type galaxies in clusters; squares indicate early-type field galaxies; and triangles indicate field spiral galaxies. Open symbols denote the two new values from the current study. The curves (courtesy G. Bryan) show the slope of the $T_{\text {blue }}$ vs. galaxy stellar mass relation depending on whether metal-poor GCs were formed at $z>7,11$, or 15 (see the text for details). 
Table 1. Basic Properties of the Target Galaxies

\begin{tabular}{ccccrrr}
\hline \hline Name & Type & $\begin{array}{c}\text { Inclination } \\
(\mathrm{deg})\end{array}$ & $\begin{array}{c}v_{\text {Helio }} \\
\left(\mathrm{km} \mathrm{s}^{-1}\right)\end{array}$ & & $\begin{array}{c}\text { Distance } \\
(\mathrm{Mpc})\end{array}$ & $M_{V}^{T}$ \\
\hline & & & & & & \\
NGC 891 & $\mathrm{Sb}$ & 84 & $831 \pm 1$ & 29.61 & 8.36 & -20.79 \\
NGC 4013 & $\mathrm{Sb}$ & 90 & $528 \pm 4$ & 30.90 & 15.1 & -20.38 \\
\hline
\end{tabular}

Note. - Morphological types are from the Hubble Atlas of Galaxies (Sandage 1961) for NGC 891 and from RC1 (de Vaucouleurs \& de Vaucouleurs 1964) for NGC 4013. Inclination and heliocentric radial velocity are from Verheijen \& Sancisi (2001) for NGC 4013. Heliocentric radial velocity for NGC 891 is from RC3 (de Vaucouleurs et al. 1991) and inclination is from Tully (1988). Distance to NGC 891 is from Tonry et al. (2001) (surface brightness fluctuations). Distance to NGC 4013 is from combining the recession velocity with respect to the $3 \mathrm{~K}$ cosmic microwave background from the NASA Extragalactic Database with $H_{0}$ $=70 \mathrm{~km} \mathrm{~s}^{-1} \mathrm{Mpc}^{-1}$. Total absolute magnitudes are from combining $V_{T}^{0}$ from RC3 with $m-M$.

Table 2. WIYN Minimosaic Observations of the Target Galaxies

\begin{tabular}{llccc}
\hline \hline \multirow{2}{*}{ Galaxy } & \multirow{2}{*}{ Date } & \multicolumn{3}{c}{ Exposure Times (s) } \\
& & $B$ & $V$ & $R$ \\
\hline \multirow{2}{*}{ NGC 891 } & 2001 Jan & $4 \times 2100$ & $3 \times 2000$ & $3 \times 1500$ \\
NGC 4013 2001 Jan & $3 \times 2100$ & $1 \times 2000$ & $3 \times 1500$ \\
& 2009 Mar & & $4 \times 2000$ & \\
\hline
\end{tabular}


Table 3. Corrected Radial Profile of the GC System of NGC 891

\begin{tabular}{lrll}
\hline \hline $\begin{array}{c}\text { Radius } \\
(\operatorname{arcmin})\end{array}$ & $\begin{array}{r}\text { Surface Density } \\
\left(\text { arcmin }^{-2}\right)\end{array}$ & Fractional Coverage & Source \\
\hline 0.48 & $9.62 \pm 3.21$ & 0.66 & HST \\
0.96 & $4.63 \pm 1.89$ & 0.43 & HST \\
1.25 & $0.64 \pm 0.57$ & 0.71 & WIYN \\
1.46 & $0.61 \pm 0.61$ & 0.36 & HST \\
1.97 & $0.43 \pm 0.43$ & 0.38 & HST \\
2.17 & $0.91 \pm 0.45$ & 0.77 & WIYN \\
2.46 & $0.00 \pm 0.00$ & 0.42 & HST \\
2.95 & $0.52 \pm 0.37$ & 0.41 & HST \\
3.16 & $0.43 \pm 0.33$ & 0.64 & WIYN \\
3.44 & $0.37 \pm 0.37$ & 0.25 & HST \\
3.94 & $0.00 \pm 0.00$ & 0.16 & HST \\
4.15 & $0.10 \pm 0.28$ & 0.47 & WIYN \\
4.45 & $0.57 \pm 0.57$ & 0.13 & HST \\
5.16 & $0.25 \pm 0.29$ & 0.43 & WIYN \\
6.12 & $-0.01 \pm 0.25$ & 0.32 & WIYN \\
7.12 & $0.09 \pm 0.30$ & 0.22 & WIYN \\
8.04 & $-0.09 \pm 0.40$ & 0.07 & WIYN \\
& & & \\
\hline
\end{tabular}

Note. - For completeness, we list all points in the radial profile made using the WIYN + HST GC candidate samples; when no GC candidates appear within a particular annulus, the surface density is written as " $0.00 \pm 0.00$ ". Negative surface densities sometimes occur because a contamination correction has been applied to each annulus. 
Table 4. Corrected Radial Profile of the GC System of NGC 4013

\begin{tabular}{lrll}
\hline \hline $\begin{array}{c}\text { Radius } \\
(\text { arcmin })\end{array}$ & $\begin{array}{r}\text { Surface Density } \\
\left(\text { arcmin }^{-2}\right)\end{array}$ & Fractional Coverage & Source \\
\hline 0.39 & $37.30 \pm 6.93$ & 1.00 & HST \\
0.82 & $9.90 \pm 2.65$ & 0.72 & HST \\
0.90 & $9.70 \pm 2.31$ & 0.72 & WIYN \\
1.34 & $7.07 \pm 2.67$ & 0.32 & HST \\
1.81 & $2.22 \pm 1.08$ & 0.89 & WIYN \\
2.78 & $0.90 \pm 0.93$ & 0.96 & WIYN \\
3.77 & $0.37 \pm 0.89$ & 0.96 & WIYN \\
4.68 & $0.36 \pm 0.90$ & 0.66 & WIYN \\
\hline
\end{tabular}

Table 5. Coefficients from Fitting Radial Profile Data

\begin{tabular}{ccccc}
\hline \hline & \multicolumn{2}{c}{ de Vaucouleurs Law } & \multicolumn{2}{c}{ Power Law } \\
\cline { 2 - 5 } Galaxy & a0 & a1 & a0 & a1 \\
\hline \multirow{2}{*}{ NGC 891 } & $3.02 \pm 0.33$ & $-2.53 \pm 0.29$ & $0.45 \pm 0.07$ & $-1.62 \pm 0.18$ \\
NGC 4013 & $4.02 \pm 0.30$ & $-3.11 \pm 0.32$ & $0.88 \pm 0.05$ & $-1.73 \pm 0.18$ \\
\hline
\end{tabular}

Table 6. Total Numbers and Specific Frequencies of the GC Systems

\begin{tabular}{crccc}
\hline \hline Galaxy & \multirow{2}{*}{$N_{G C}$} & $S_{N}$ & $T$ & $T_{\text {blue }}$ \\
\hline NGC 891 & $70 \pm 20$ & $0.3 \pm 0.1$ & $0.6 \pm 0.3$ & $0.3 \pm 0.2$ \\
NGC 4013 & $140 \pm 20$ & $1.0 \pm 0.2$ & $1.9 \pm 0.5$ & $1.4 \pm 0.3$ \\
\hline
\end{tabular}

